ARTICLE

\title{
MORC1 represses transposable elements in the mouse male germline
}

William A. Pastor ${ }^{1, \star}$, Hume Stroud ${ }^{1, *, \dagger}$, Kevin Nee $^{1}$, Wanlu Liu ${ }^{1}$, Dubravka Pezic ${ }^{2}$, Sergei Manakov ${ }^{2}$, Serena A. Lee ${ }^{1}$, Guillaume Moissiard', Natasha Zamudio ${ }^{3}$, Déborah Bourc'his ${ }^{3}$, Alexei A. Aravin², Amander T. Clark ${ }^{1,4} \&$ Steven E. Jacobsen ${ }^{1,4,5}$

The Microrchidia (Morc) family of GHKL ATPases are present in a wide variety of prokaryotic and eukaryotic organisms but are of largely unknown function. Genetic screens in Arabidopsis thaliana have identified Morc genes as important repressors of transposons and other DNA-methylated and silent genes. MORC1-deficient mice were previously found to display male-specific germ cell loss and infertility. Here we show that MORC1 is responsible for transposon repression in the male germline in a pattern that is similar to that observed for germ cells deficient for the DNA methyltransferase homologue DNMT3L. Morc1 mutants show highly localized defects in the establishment of DNA methylation at specific classes of transposons, and this is associated with failed transposon silencing at these sites. Our results identify MORC1 as an important new regulator of the epigenetic landscape of male germ cells during the period of global de novo methylation.

\footnotetext{
${ }^{1}$ Department of Molecular, Cell and Developmental Biology, University of California Los Angeles, 4028 Terasaki Life Sciences Building, 610 Charles E. Young Drive East, Los Angeles, California 90095, USA. ${ }^{2}$ Division of Biology and Biochemical Engineering, California Institute of Technology, 1200 E California Boulevard, Pasadena, California 91125, USA. ${ }^{3}$ Unité de génétique et biologie du développement, Instititute Curie, CNRS UMR3215, INSERM U934, Paris 75005, France. ${ }^{4}$ Eli and Edythe Broad Center of Regenerative Medicine and Stem Cell Research, University of California Los Angeles, 615 Charles E. Young Drive South, Los Angeles, California 90095, USA. ${ }^{5}$ Howard Hughes Medical Institute, University of California Los Angeles, 675 Charles E. Young Drive South, Los Angeles, California 90095, USA. * These authors contributed equally to this work. †Present address: Department of Neurobiology, Harvard Medical School, 220 Longwood Avenue, Boston, Massachusetts 02115 USA. Correspondence and requests for materials should be addressed to A.T.C. (email: clarka@ucla.edu) or to S.E.J. (email: jacobsen@ucla.edu).
} 
T wo Morc genes in A. thaliana, AtMorc1 and AtMorc6, were identified in forward genetic screens for novel transcriptional repressors ${ }^{1,2}$. AtMORC1 and AtMORC6 are required for silencing of a variety of transposons and are essential for higher-order chromatin compaction. The single Morc gene in Caenorhabditis elegans was also shown to be required for silencing of a repetitive transgene locus ${ }^{1}$. The founding member of the Morc gene family is mammalian Morc1. MORC1 is highly expressed in the blastocyst and male germline but is not expressed in most differentiated cells ${ }^{3}$. Mice deficient for MORC1 are normal, except that homozygous mutant males are infertile with small testicles (hence the name microrchidia) ${ }^{4,5}$. Male germ cells in the Morc1 mutant do not undergo successful chromosomal pairing during the zygotene stage of meiosis and instead undergo apoptosis, with no germ cells surviving to complete prophase I.

During germ cell development, most DNA methylation is lost between E8.5 and E13.5. Then, between E13.5 and birth ( E19), the genome undergoes global de novo methylation ${ }^{6-8}$. Failure to establish DNA methylation at this time causes transposon upregulation and meiotic failure. Indeed, the meiotic block in the Morc1 mutant is similar to that observed for mice that have defects in DNA methylation and transposon repression, including mice deficient for DNA methyltransferases ${ }^{9-11}$ or the pre-meiotic Piwi-interacting RNA (piRNA) pathway ${ }^{12,13}$. Therefore, we hypothesized that MORC1 might be a critical factor for transposon silencing and DNA methylation in the mouse germline. Here we demonstrate that MORC1-deficient male germ cells undergo transposon derepression starting in late embryogenesis and continuing through the onset of meiosis. We also demonstrate that this phenotype is associated with failed locus-specific de novo methylation targeted specifically towards late-methylating transposon sequences.

\section{Results}

MORC1 represses transposons in the male germline. To further characterize MORC1 we used a previously described $\mathrm{FVB} / \mathrm{N}$ Morcl mutant $\left(\mathrm{Morcl}^{\text {tg }}\right)$ mouse strain in which a tyrosinase gene was integrated into the Morcl locus ${ }^{5}$. Transgene insertion resulted in loss of exons 2-4, eliminating a large region of the GHKL ATPase domain including residues predicted to be critical for catalysis and ATP binding ${ }^{14}$ (Fig. 1a). Consistent with previous reports, we found that Morctg/tg mice have a spermatogenesis defect with a complete absence of post-meiotic spermatids and spermatozoa (Supplementary Fig. 1a-c).

Quantative reverse transcription-PCR (qRT-PCR) from wild type (WT), embryonic whole testis indicates that Morcl messenger RNA becomes detectable at E14.5 and peaks at E16.5 (Supplementary Fig. 2a), which intriguingly is a period of rapid transposon methylation in the male germline. We generated an antibody against the coiled-coil domain of mouse MORC1 (Supplementary Fig. 2b) and found that MORC1 was localized to the nucleus of male germ cells at E16.5 (Fig. 1b). Conversely, MORC1 protein was undetectable in Morc1 ${ }^{\text {tg/g }}$ mutant germ cells (Fig. 1b). To test for transposon derepression in Morcltg/tg germ cells, we performed immunostaining for LINE1 ORF1p and intracisternal particle A (IAP) at postnatal day 14.5 (P14.5). We found that both of these transposon classes were ectopically expressed during early postnatal development, with particular enrichment of LINE1 ORF1p in the meiotic cells towards the centre of the tubule (Fig. 1c,d). LINE1 ORF1p was also derepressed at E16.5 and E18.5, showing that transposon derepression in Morc1 $1^{\text {tg/tg }}$ arises well before the apparent meiotic defect (Supplementary Fig. 2c).

To identify which genes and specific transposons are targets of MORC1-mediated repression, we performed mRNA-Sequencing
(RNA-Seq) of whole testes from Morc1 $1^{\text {tg/tg }}$ and Morc1 ${ }^{\text {tg } /+}$ heterozygous controls at P10.5 and P14.5. Given that germ cells make up only a small percentage of the testis during the embryonic early and postnatal period, we also performed RNASeq on ribosomal RNA-depleted total RNA from sorted germ cells at E16.5, E18.5, P2.5 and P10.5. To purify germ cells, we crossed the Morc1 ${ }^{t g}$ allele into the Oct4-IRES-eGfp reporter strain, which exhibits a distinct $\mathrm{eGFP}^{+}$population from e9.5 to $\mathrm{P} 2.5$ (Supplementary Fig. $3 a^{15,16}$. To isolate germ cells at P10.5, we gated the side scatter (SSC) low (SSC ${ }^{\mathrm{Lo}}$ ), epithelial cell adhesion molecule (EPCAM) high $\left(\mathrm{EPCAM}^{\mathrm{Hi}}\right)$ and major histocompatibility complex negative $\left(\mathrm{MHCI}^{-}\right)$population of testicular cells (Supplementary Fig. 3b). A list of sequencing samples and the experiments to which they correspond is included in Supplementary Data 1. Consistent with RT-PCR and published data ${ }^{5,17}$, Morcl mRNA was confirmed to show high expression at E16.5 and lower expression at later time points in the sorted germ cells (Fig. 2a).

The RNA-Seq data showed a broad transposon derepression defect in Morct $1^{\text {tg/tg }}$ starting at E16.5 (Fig. 2c-e and Supplementary Data 2). Quantitative RT-PCR analysis of various transposable element classes gave similar results as RNA-Seq data (Supplementary Fig. 4a). In addition, RNA-Seq on sorted Morc1tg/tg and control germ cells at P10.5 resembled the pattern of transposon derepression observed in whole testes (Supplementary Fig. 4a). Heterozygous Morc1 $1^{\text {tg }}++$ mice showed no marked increase in transposon expression relative to WT Morcl ${ }^{+/+}$mice, confirming their validity as littermate controls (Supplementary Fig. 4b).

Different transposon classes showed different patterns of

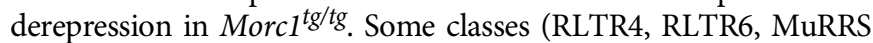
and Etn) were upregulated during embryogenesis but silenced even in the knockout at later time points (Fig. 2b). Other transposons (MMERVK10C, GLN and some IAP species) were most highly upregulated at postnatal time points (Fig. 2c,d). LINEs were upregulated both in late embryogenesis and again at P14.5 after the onset of meiosis (Fig. 2e), which was confirmed by immunofluorescence (Fig. 1d and Supplementary Fig. 2c). These fluctuations in transposon upregulation may reflect differences in the inherent transcriptional programmes of certain transposon classes, as well as varied effectiveness of other, partially redundant transposon repression pathways at different times. In the aggregate, however, these results indicate that MORC1 constitutes a new participant in transposon repression in the mammalian germline, acting on many different elements. Notably, MORC1 silences many transposon classes well after it is downregulated, consistent with it acting through an epigenetic mark such as DNA methylation.

piRNA biogenesis occurs normally in the Morcl mutant. During this period in germline development (E14.5 to birth), germ cells undergo mitotic arrest and global nuclear reprogramming that most notably involves genome-wide de novo DNA methylation mediated by the DNMT3A/DNMT3L complex ${ }^{11,18}$. The premeiotic piRNA pathway, involving the nuclear PIWI protein MIWI2, is also active during this period in promoting transposon silencing. To evaluate whether MORC1 acts on the same transposon classes as DNMT3L or MIWI2, we performed RNASeq on whole testes from Dnmt3l-/- (ref. 10) and Miwi2 ${ }^{-/-}$ (ref. 12) mice and their respective controls at P10.5, and compared this with the Morclg/tg P10.5 whole testis data set. Morcl ${ }^{\text {tg/tg }}$ and Dnmt3l ${ }^{-1-}$ exhibited derepression of an overlapping set of transposons, primarily long terminal repeat (LTR) retrotransposons, while the Miwi2 ${ }^{-1-}$ mutant testes had a milder phenotype and showed derepression of specific LINE elements and the IAP-Ey class of retrotransposons, which was not affected in Morcl ${ }^{t g / t g}$ or Dnmt3l ${ }^{-/-}$mutant testes (Fig. 2f-h). 
a 1

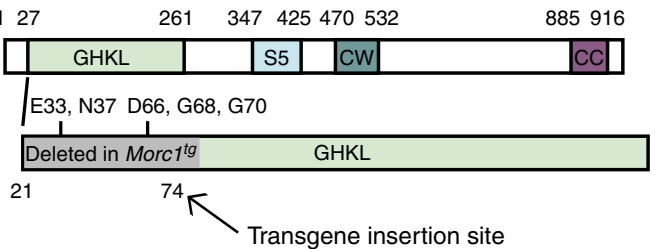

b

Morc1 het E16.5

DAPI

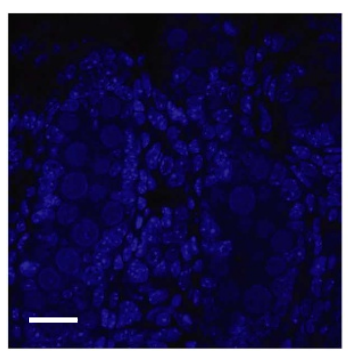

Morc1 KO E16.5

DAPI

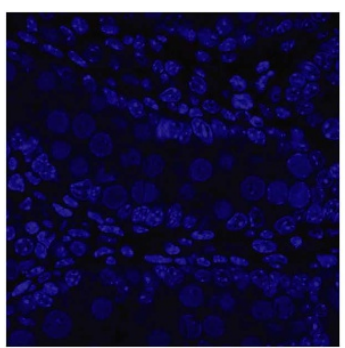

C

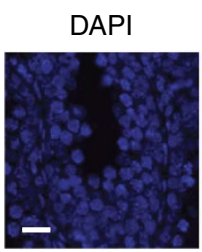

DAPI

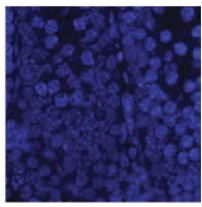

P14.5 Morc1 WT

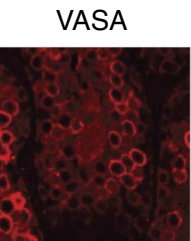

VASA

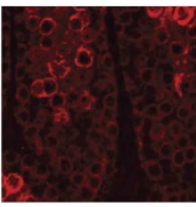

VASA

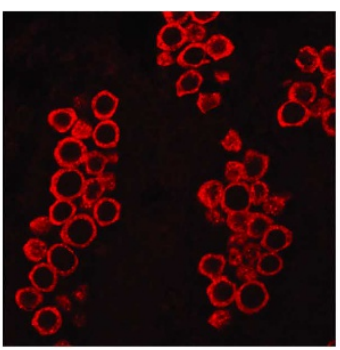

VASA

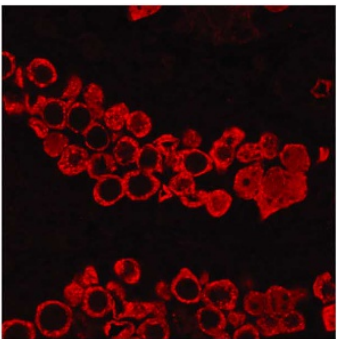

IAP GAG

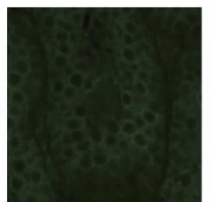

LINE1 ORF1p

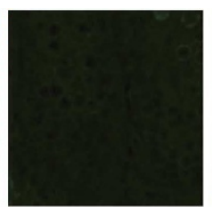

MORC1

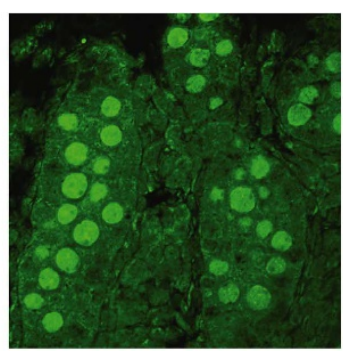

MORC1

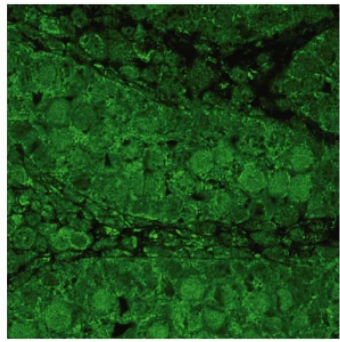

d

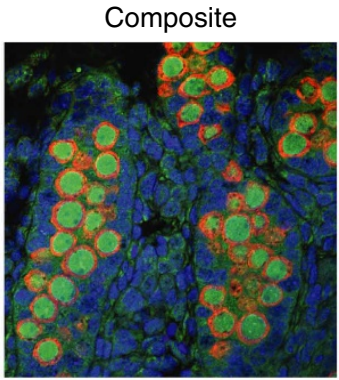

Composite

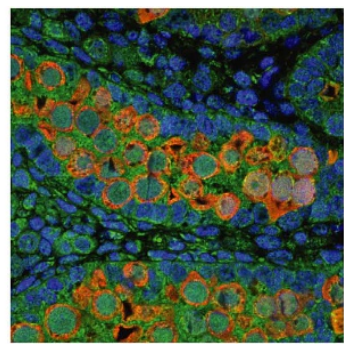

P14.5 Morc1 KO

Figure 1 | MORC1 is a nuclear protein essential for transposon repression. (a) Domain structure of Morc1 gene and disruption in Morct ${ }^{t g}$ allele.

Deleted residues predicted to be critical for catalytic activity or ATP binding are denoted. (b) Detection of MORC1 by immunofluorescence (IF) in E16.5 testes. MORC1 is present as a germ cell-specific nuclear protein in the Morc $7^{\text {tg } /+}$ (het) control but is absent from the Morc $7^{\text {tg/tg }}$ (knockout (KO)). Aberrant expression of IAP GAG (c) and LINE ORF1p (d) in Morc $7^{t g} / \mathrm{tg}$ as detected by IF at P14.5. Note that IAP is overexpressed in most germ cells, whereas LINE is primarily present in the more differentiated cells deeper into the tubule. Scale bars, $20 \mu \mathrm{m}$ (b-d).

The lack of overlap between Morc1 and Miwi2 predicts that piRNAs would be unperturbed in Morc1 $1^{\text {tg/tg }}$ mice. To test this, we performed small RNA sequencing of the testis at E16.5 to examine piRNA production. Our data revealed that the ratio of piRNA/microRNA and the generation of antisense piRNAs were unaltered in Morcl $^{\text {tg/gg }}$ (Table 1), indicating that the piRNA pathway remains largely intact in Morc1 $1^{t g / t g}$ testis at E16.5, and that transposon derepression in Morcltg/tg is most likely to be independent of the piRNA pathway. In fact, at P10.5 we observed an increase in the fraction of piRNAs derived from LTR retrotransposons, especially of the IAP family, in the MORC1-deficient testis (Supplementary Fig. 5a-c), similar to that observed in Dnmt3l ${ }^{-/-19}$. These LTR transposon-derived piRNAs corresponded to primary sense piRNAs (Supplementary Fig. 5d,e), suggesting that they are probably more abundant simply because the underlying mRNA species are derepressed in Morcltg/tg, and some fraction are converted to piRNAs (Supplementary Fig. 5f). Hence, our results indicate that MORC1 acts in a transposon-silencing pathway independent of piRNA production. 

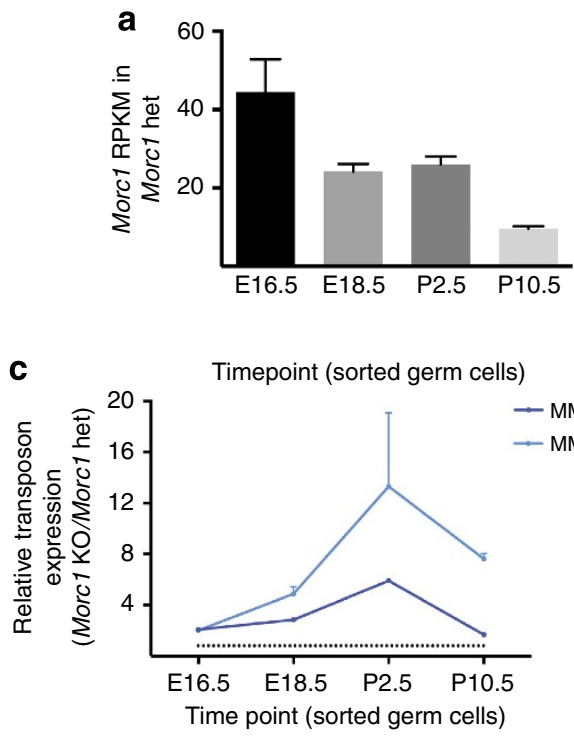

e

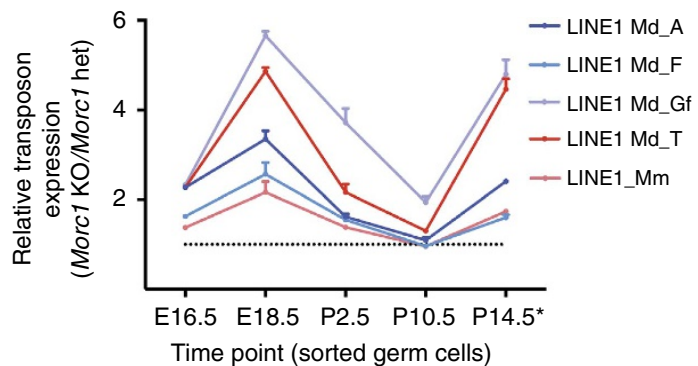

*Whole testis

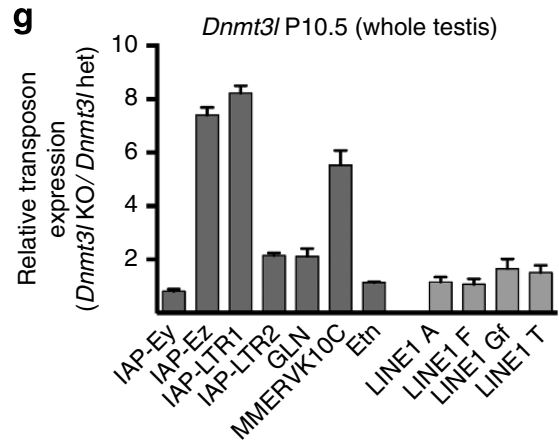

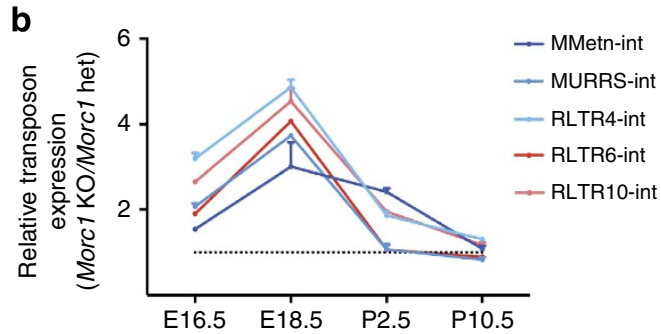

d

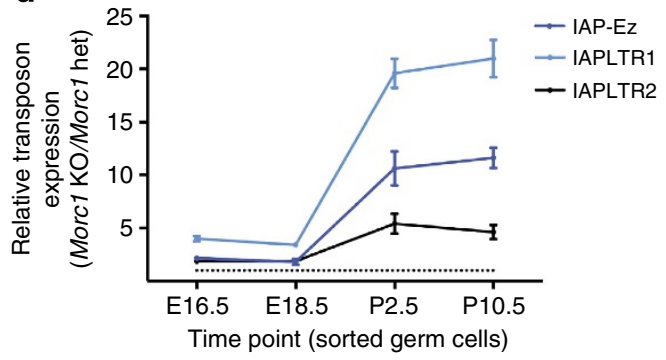

f
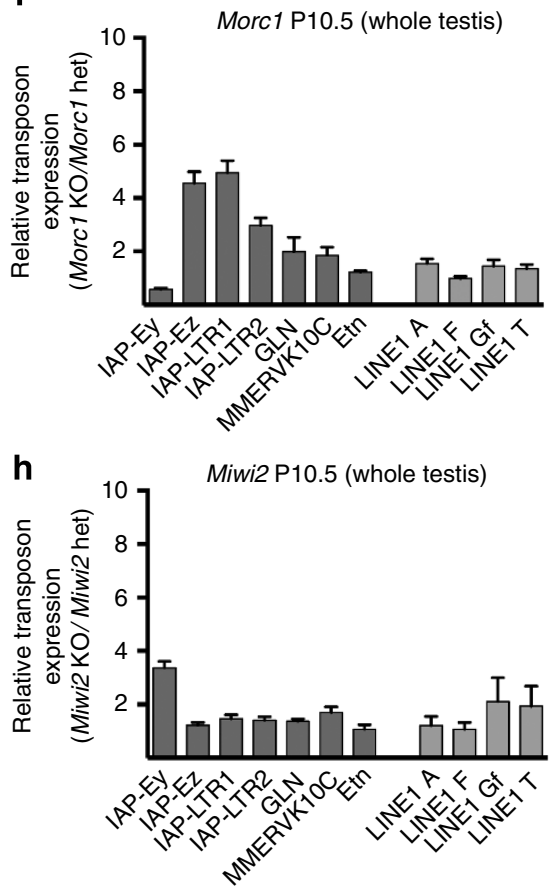

Figure 2 | Morc1 ${ }^{\text {tg/tg }}$ shows transposon upregulation resembling Dnmt31 /- $^{-}$. (a) Expression of Morc1 mRNA over development in Morc $7^{\text {tg } /+}$, as measured by RNA-seq. (b-e) Overexpression of transposon species over the course of mammalian development, represented as a ratio of expression in the Morc $7^{\text {tg } / \mathrm{tg}}$ and the Morctg/ + control. Some LTR transposons show upregulation selectively in late embryogenesis (b), while others are overexpressed postnatally (c,d), and LINE elements are overexpressed both during late embryogenesis and again at the onset of meiosis (e). Overexpression of transposons in MORC1- (f), DNMT3L- (g) and MIWI2- (h) deficient whole testis. For a-e, the dotted line indicates a fold change of one. For a-h. Two to four replicates per genotype were analysed; all data are RNA-seq from sorted germ cells or whole testis as indicated. Mean + s.e. plotted.

\section{Table 1 | piRNA abundance and characteristics in E16.5

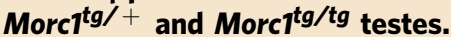

\begin{tabular}{lcc} 
& Morc1 het & Morc1 Ko \\
\hline Putative piRNA/miRNA & 0.55 & 0.59 \\
Sense/antisense & 1.34 & 1.34 \\
Primary/secondary & 4.43 & 3.91
\end{tabular}

miRNA, micro RNA; piRNA, Piwi-interacting RNA; smRNA, small RNA.

Ratios of putative piRNA/miRNA, sense piRNA/antisense piRNA and primary piRNA/secondary piRNA populations are indicated for smRNA obtained from pooled E16.5 Morctg/ + and Morct $7^{t / t g}$ testes. No substantial defect in piRNA biogenesis is observed in MORC1-deficient testis.
Hypomethylation of transposable elements in Morc1 ${ }^{\text {tg/tg }}$ Because of the resemblance between transposons derepressed in Morc1 ${ }^{\text {tg/tg }}$ and Dnmt3l ${ }^{-/-}$testes (Fig. 2f,g), we sought to examine whether Morcl might affect global DNA methylation levels. To address this, we performed whole genome bisulfite sequencing at E16.5, P2.5 and P10.5 on sorted Morc1 ${ }^{\text {tg/tg }}$ and control germ cells isolated as above. At E16.5, the germline is undergoing de novo DNA methylation and by $\mathrm{P} 2.5$ de novo methylation is largely complete. Between roughly P2.5 and P10.5, germline cells re-enter the cell cycle and either initiate the first wave of spermatogenesis to generate meiotic cells or localize to the basement membrane and generate the long-term 
self-renewing spermatogonial stem cell population ${ }^{20,21}$. In contrast to Dnmt3l ${ }^{-I^{-}}$mutant germ cells that show a dramatic global reduction in DNA methylation ${ }^{22}$, we found no change in global levels of methylation at any time point in Morcl'tg/tg-sorted germ cells (Fig. 3a). Thus, despite the similar morphological phenotypes and transposon expression defects of Morc1 $1^{\text {tg/tg }}$ and Dnmt3l ${ }^{-1-}$ mice, MORC1 does not act by controlling de novo or maintenance methylation at a genomewide level.

In mammals, DNA methylation is very dynamic and promoter DNA methylation frequently correlates with gene repression. To determine whether there may be localized defects in DNA methylation in Morc1 $1^{\text {tg/tg }}$, and whether these are associated with derepressed transposons identified by RNA-Seq, we calculated statistically significant differentially methylated regions (DMRs) in the Morcl $1^{\text {tg/tg }}$ germ cells relative to the Morcl $1^{\text {tg/ }}+$ control. At E16.5, we found very few DMRs (Fig. 3b). However, at P2.5 we identified 6,309 hypomethylated regions (Supplementary Data 3) but only 145 hypermethylated regions (Supplementary Data 4), indicating that Morc1 ${ }^{\text {tg/tg }}$ germ cells have locus and stage-specific DNA methylation defects (Fig. 3b). In addition, the overwhelming majority of regions identified as hypomethylated at P2.5 remain hypomethylated at P10.5 (Fig. 3c) and only a few regions lost methylation between P2.5 and P10.5 (Fig. 3d).

The hypomethylated DMRs in Morcl ${ }^{\text {tg/tg }}$ germ cells were highly enriched for LINE and LTR transposons rather than protein-coding genes compared with control regions (see Supplementary Methods), consistent with the transposon expression defects observed in Morcl ${ }^{\text {tg/tg }}$ germ cells (Fig. 4a). Indeed,
93.9\% of hypomethylated DMRs contained an LTR or LINE, compared with $40.6 \%$ of control DMRs. The hypomethylated DMRs were strongly concentrated in the categories of transposons that showed evidence of derepression (Fig. 4b-d) during some stage of development before meiosis.

A partial exception to this trend were IAP elements. Hypomethylated DMRs were strongly enriched for IAP elements and corresponding LTRs (Supplementary Fig. 6a), but there was a poor correspondence between the extent to which a subcategory of IAPs was upregulated and the frequency of overlap with DMRs (Fig. 2d and Supplementary Fig. 6b). This is probably because certain highly similar repetitive elements such as LTR1 give very few uniquely mapping reads and are therefore missing from the data set. To overcome this, we also mapped the BS-seq data to RepBase consensus sequences for relevant transposons. We confirmed hypomethylation of the upregulated IAPLTR1 class (Supplementary Fig. 6c). Mapping to repeat consensus sequences also confirmed hypomethylation of LINE and LTR classes, which frequently overlap with DMRs (Fig. 4c-e).

Only 20 protein-coding genes contained an annotated transcription start site (TSS) within $1 \mathrm{~kb}$ of a hypomethylated DMR (Supplementary Data 5) and only 3 contained a TSS within a DMR. Interestingly, all three of these genes (Nebulin, Tmc2 and Cdkl4) contain an RLTR10A transposable element immediately upstream of the TSS and all three genes showed a statistically significant increase in expression (Supplementary Fig. 7 and Supplementary Data 5). Thus, at a very few loci, MORC1 regulates genic expression, probably as a byproduct of its transposon repression activity in the local neighbourhood.
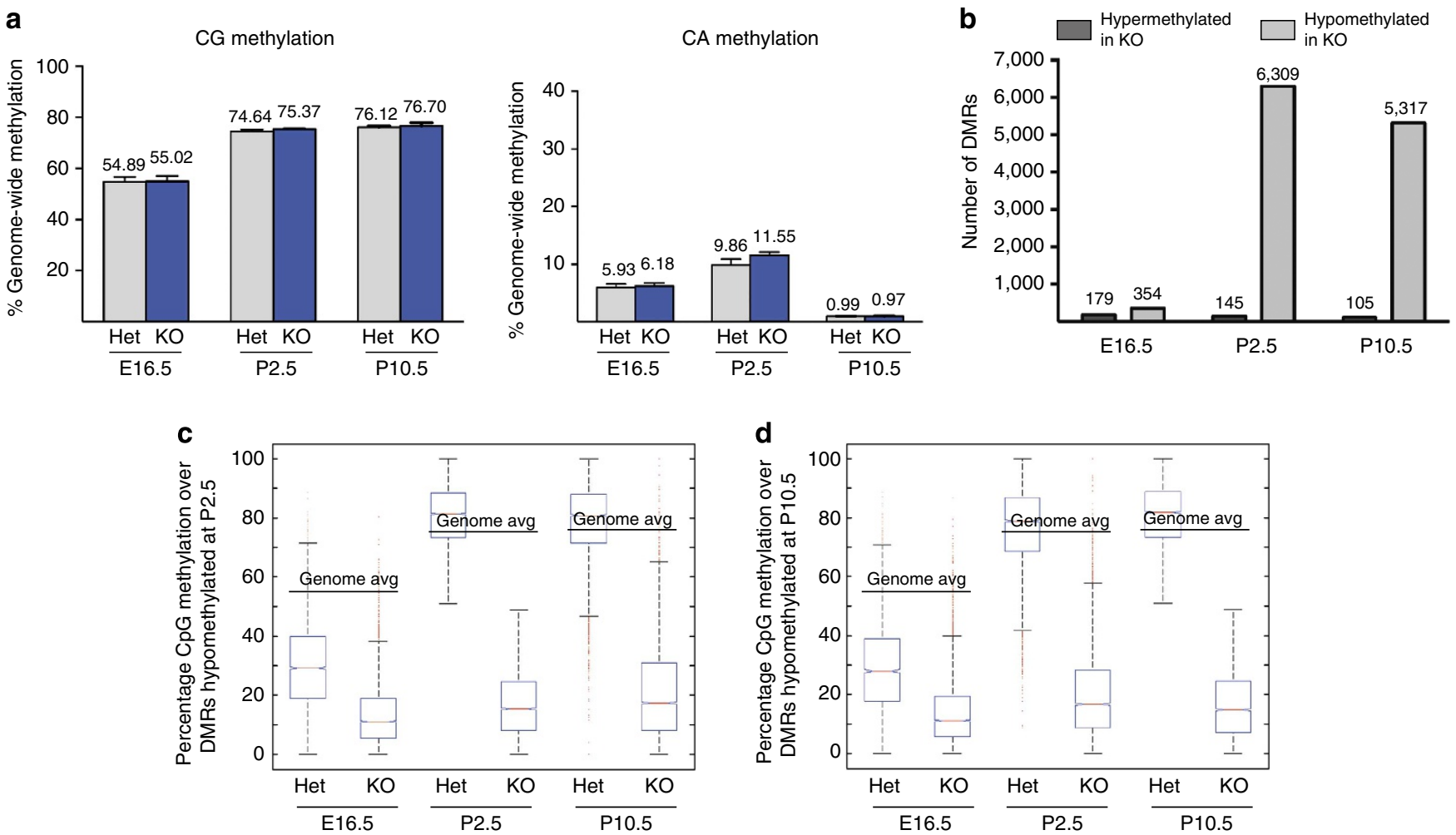

Figure 3 | MORC1 regulates DNA methylation in a stage- and locus-specific manner. (a) Total CG and CA methylation levels in Morct ${ }^{\text {tg } /+}$ and Morc $7^{t g} / t_{g}$ sorted germ cells. Three to four replicates per genotype and time point were analysed; mean and s.e. are indicated. (b) Number of hypermethylated and hypomethylated DMRs, calculated using pooled BS-seq data at each time point. (c) Boxplots of DNA methylation levels at E16.5, P2.5 and P10.5 are shown for the set of regions that were computed as hypomethylated at P2.5 in Morctg/tg germ cells. (d) Box plots of levels of DNA methylation levels in germ cells at E16.5, P2.5 and P10.5 are shown for the set of regions that were computed as hypomethylated at P10.5 in Morctg/tg. For $\mathbf{c}, \mathbf{d}$, each DMR constitutes one point in each box plot. Red lines, median; edges of boxes, 75th (top) and 25th (bottom) percentiles; whiskers, minimum and maximum points within $1.5 \times$ the interquartile range; red dots, outliers. 
a

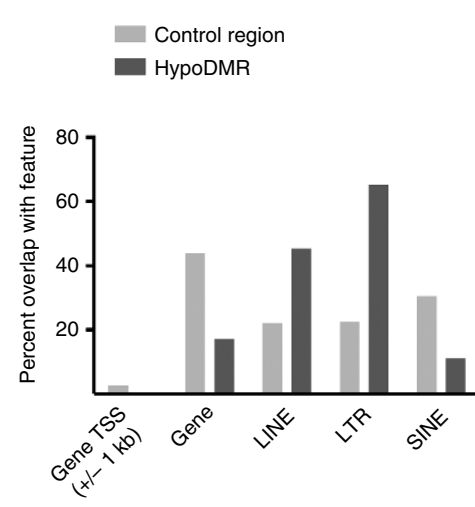

C
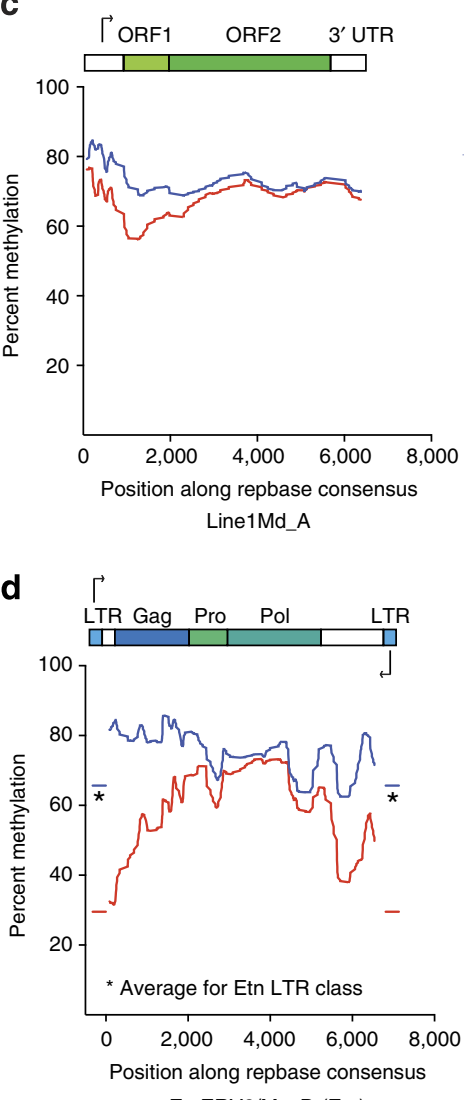

EtnERV2/MusD (Etn) b

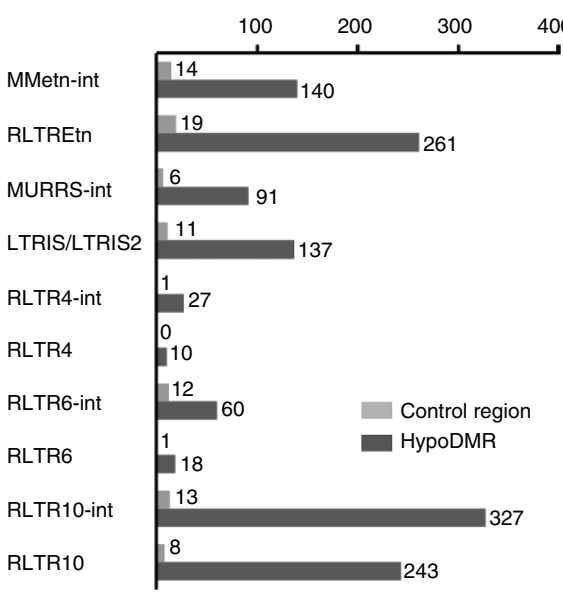

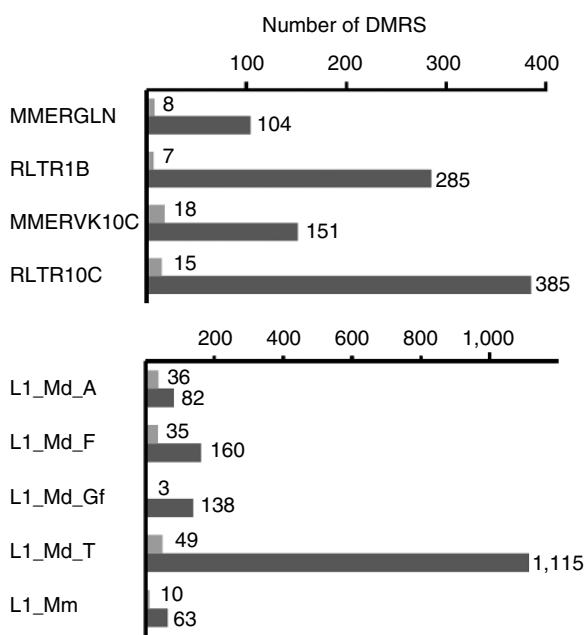

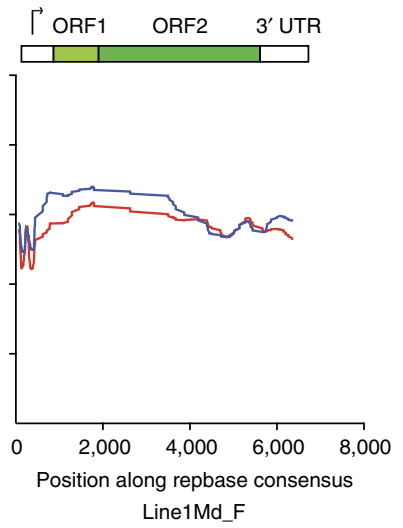

Line1Md_F

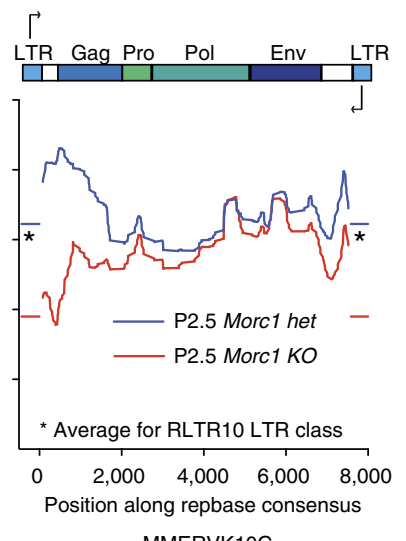

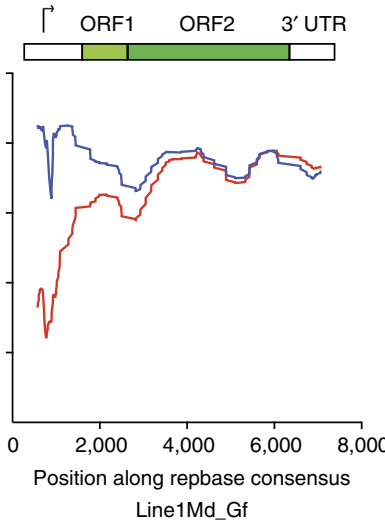

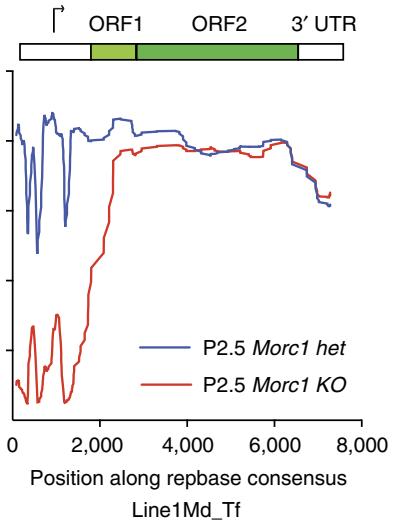

e

Figure 4 | Hypomethylated regions in Morcitg/tg correspond to upregulated transposon classes. (a) The overlap of hypomethylated DMRs and control regions (randomly selected regions whose methylation is unaffected by loss of MORC1) with genes and transposon classes is indicated. A DMR or control is counted as overlapping with a feature if there was at least one basepair overlap. (b) Overlap of hypomethylated DMRs with transposon classes upregulated in Morct ${ }^{\mathrm{tg}} / \mathrm{tg}$. Metaplot of methylation over LINE (c) and LTR (d) retrotransposons. BS-seq data were mapped to annotated RepBase consensus sequences for each transposon class and methylation is plotted at each CG in the annotated RepBase consensus sequence relative to the consensus sequence. The first 50 bases from each element, which often have low read coverage, are omitted. As it was usually not possible to determine the orientation of LTR-derived reads relative to LTR transposons, the average methylation level for the relevant LTR species is shown. (e) Global hypomethylation of LTR species corresponding to upregulated transposon classes. Again, BS-seq data were mapped to RepBase consensus sequence for these LTRs.

Considering MORC1's role as local modulator of DNA methylation, we examined changes in methylation in the three well-characterized paternally methylated imprinted loci ${ }^{23}$. Methylation occurred normally at two of the three loci (H19 and Dlk1-Gtl2), but the imprinting control region of
Rasgrf1 showed increased transcription and hypomethylation in the Morc1 mutant (Supplementary Fig. 8). Interestingly, this is a transposon-rich area, which has previously been demonstrated as a target of the piRNA pathway ${ }^{24}$ (see Discussion below). 
Of the very few hypermethylated loci observed in the Morc1 mutant, most were not conserved across time points and are probably a consequence of biological or statistical noise. However, 15 hypermethylated DMRs were reproducible between P2.5 and P10.5. Nine of these 15 were embedded in 2 transcripts upregulated in Morc1 ${ }^{\text {tg/tg: }} 6$ DMRs contained within the body of the Cdkl4 gene described above and 3 DMRs in an unannotated transcript probably originating from a hypomethylated IAPLTR1 element (Supplementary Fig. 9). These are probably examples of transcriptional run-through from a nearby promoter causing methylation of a locus, a phenomenon that has been described for some imprinted loci ${ }^{25}$.

DMRs are sites of transposon transcriptional initiation. The highly localized affect of MORC1 on the germline epigenome suggests that MORC1 may function at the transcriptional start sites of transposons to facilitate their silencing and methylation. In support of this, we discovered that hypomethylation in Morc $1^{\text {tg/tg }}$ mutant germline cells was concentrated at the $5^{\prime}$ - ends of LINE elements coincident with the location of transcriptional initiation (Fig. 4c) ${ }^{26}$. Furthermore, LTR transposons, which are typically flanked by LTRs that serve promoter and enhancer functions ${ }^{27}$, showed hypomethylation on both ends in Morcl ${ }^{\mathrm{tg} / \mathrm{tg}}$ (Fig. 4d), and the LTRs themselves are heavily hypomethylated (Fig. 4d,e).

We also noted that hypomethylated DMRs in $\mathrm{Morcl}^{\mathrm{tg} / \mathrm{tg}}$ germ cells were late targets for de novo methylation during the course of epigenetic reprogramming, since in control Morc1 ${ }^{\text {tg } /+}$ cells these genomic regions were also hypomethylated relative to the genome average at E16.5 (Fig. 3c,d and Supplementary Fig. 10). This suggests that these loci are somewhat resistant to de novo methylation. Consistent with this possibility, we also discovered that these Morcl affected genomic regions have increased $\mathrm{H} 3 \mathrm{~K} 4 \mathrm{me} 3$ relative to control regions of the genome in WT cells E13.5 (Supplementary Fig. 11a) ${ }^{28}$. To determine whether these loci are enriched in H3K4me3 during the dynamic de novo methylation of the germline genome, we performed chromatin immunoprecipitation (ChIP) analysis for $\mathrm{H} 3 \mathrm{~K} 4 \mathrm{me} 3$ at E16.5 and confirmed that these regions still exhibited higher H3K4me3 (Fig. 5a). In contrast, other chromatin marks such as H2B10ac, K3K27ac and H3K27me3 showed no correlation with Morc1 $1^{\text {tg/tg }}$ DMRs (Supplementary Fig. 11b). It is well established that $\mathrm{H} 3 \mathrm{~K} 4$ methylation antagonizes de novo DNA methylation by blocking DNMT3A/ 3L binding to histone $\mathrm{H} 3$ (ref. 29). Thus, the presence of $\mathrm{H} 3 \mathrm{~K} 4 \mathrm{me} 3$ could potentially explain why these loci methylate with slow kinetics and require an additional factor (MORC1) for eventual silencing and methylation. It is also well established that $\mathrm{H} 3 \mathrm{~K} 4 \mathrm{me} 3$ is a mark of transcriptional start sites, consistent with the idea that many DMRs are TSSs for transposons that are active in the embryonic germline.

At E16.5, we found that RNA transcripts were significantly elevated at Morc1 ${ }^{\text {tg/tg}}$-hypomethylated DMRs relative to the surrounding areas, even in control Morc1 $1^{\text {tg/ }}+$ germ cells (Fig. 5b). However, by P10.5, this RNA expression was severely repressed in control Morcl ${ }^{t g /+}$ germ cells with modest but increased expression in Morcl ${ }^{\text {tg/tg }}$ (Fig. 5b). This data is consistent with a model in which the hypomethylated DMRs correspond to TSSs that are normally methylated and suppressed during development.

To further confirm that these DMRs correspond to transcriptional start sites, we employed ATAC-seq, which can be used to identify areas of open chromatin that are a signature of promoter and enhancer sites ${ }^{30}$. We confirmed that ATAC-seq can be accurately adopted for small sample sizes, that reads cluster near transcriptional start sites in E16.5 germ cells, and that ATAC-seq read density at the TSS correlates with gene expression (Supplementary Fig. 12). We found that ATAC-seq peaks overlapped tightly with DMRs (Fig. 5c,d) and most DMRs showed ATAC-seq reads substantially elevated over background (Fig. 5e). In contrast, reads from the naked DNA control were not enriched over DMRs (Fig. 5d). At P10.5, a more limited subset of DMRs exhibited elevated ATAC-seq reads (Fig. 5e), consistent with the observation that transcription is retained only at some DMRs in postnatal germ cells (Supplementary Fig. 13a,b). Other sites lose ATAC peaks and transcription (Supplementary Fig. 13c), either because relevant transcription factors are absent or because other mechanisms of transposon silencing are effective. Importantly, at E16.5, where we observe expression from DMR regions in both Morc1 ${ }^{\text {tg/ }+}$ and Morc1 $1^{\text {tg/tg }}$ cells, we also observed a high ATAC-seq signal in both Morc1 ${ }^{\text {tg/ }}+$ and Morc1 ${ }^{\text {tg/tg }}$ cells (Fig. 5d). In contrast, at P10.5, where DMRs are silenced in heterozygotes but remain expressed in $M o r c 1^{t g / t g}$, we only observed high ATAC-seq signal in the Morc1 ${ }^{\text {tg/tg }}$ cells (Fig. 5d). These results support the view that DMRs in Morc1 ${ }^{t g / t g}$ cells correspond to promoters of transposons that fail to silence properly, leading to an inappropriately open chromatin state, and ectopic transposon expression, which is retained at P10.5, even after MORC1 expression has ceased.

\section{Discussion}

The results of this study identify MORC1 as a critical regulator of transposon repression in the male germline. MORC1 does not act as a global regulator of DNA methylation. Instead, MORC1 functions to facilitate DNA methylation of a variety of transposons in the germline with very little effect on the expression or methylation of protein-coding genes. The observation that Morc homologues are required for gene silencing in Arabidopsis, C. elegans and now mammals suggests that the Morc family of proteins constitute conserved epigenetic regulators that probably function in a wide variety of eukaryotic organisms and developmental contexts.

The Morcl ${ }^{\operatorname{tg} / \mathrm{tg}}$ phenotype of transposon derepression and a block in meiosis prophase I superficially resembles the phenotype observed in mice deficient for proteins involved in the prepachytene piRNA pathway, including Mili ${ }^{31,32}$, Miwi2 (ref. 12), MitoPLD ${ }^{33}$, Mov10L1 (refs 34,35), Mael ${ }^{36,37}, \mathrm{Tdrkh}^{38}$, Tdrd9 (ref. 13) and $M V H^{39,40}$. What distinguishes $M o r c 1^{\text {tg/tg }}$ from these characterized pre-pachytene piRNA mutants, however, is the apparently normal piRNA biogenesis in Morc1 ${ }^{\text {tg/tg }}$ (Table 1). We do note similarities in the pattern of hypomethylation in Morc1 ${ }^{\text {tg/tg }}$ and $\mathrm{Mili}^{-/-}$mutant germ cells, including the Rasgrf1 imprinting control region ${ }^{24}$, as well as many of the same transposon families ${ }^{41}$. The dissimilarity in transposon repression observed in Miwi2 ${ }^{-/-}$and Morc1 ${ }^{\text {tg/tg }}$ germ cells (Fig. 2f,h) suggests that MORC1's role in the nucleus is independent from the nuclear piRNA pathway mediated by MIWI2. It is possible that MORC1 participates downstream of the nuclear piRNA pathway during embryogenesis and has a separate, piRNAindependent silencing role during the postnatal stages. This could cause Morcl $^{\text {tg/tg }}$ to have a broader transposon derepression phenotype than Miwi2-/- Alternatively, there may exist a MILI-dependent, MIWI2-independent mechanism for promoting methylation of target loci.

TEX19.1 has also been implicated in transposon repression in the male germline and has no known link to the piRNA pathway ${ }^{42}$. However, TEX19.1 is cytoplasmic $^{42,43}$, shows dysregulation only of MMERVK10C elements ${ }^{42,44}$ and Tex $19.1^{-/-}$has an incomplete infertility defect ${ }^{42,43}$. Thus, the Morc1 $1^{\text {tg/tg }}$ and Tex19.1 ${ }^{-1-}$ defects are fairly dissimilar and there is no evidence that they participate in the same pathway. 
a

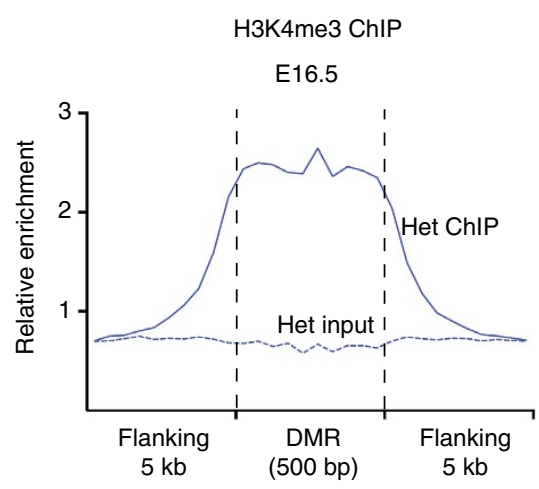

b

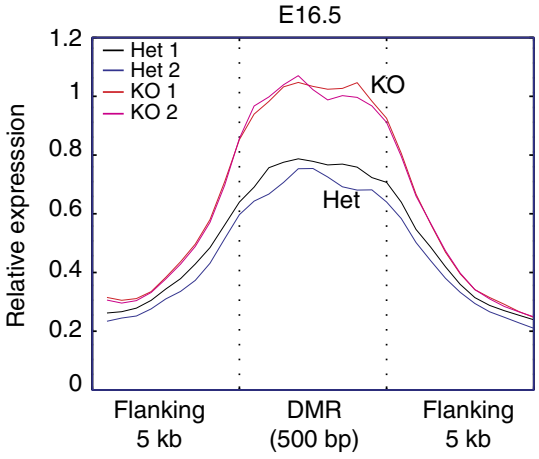

RNAseq reads (sorted germ cells)

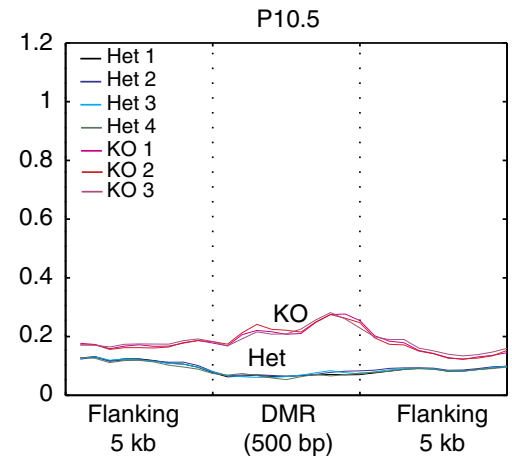

C

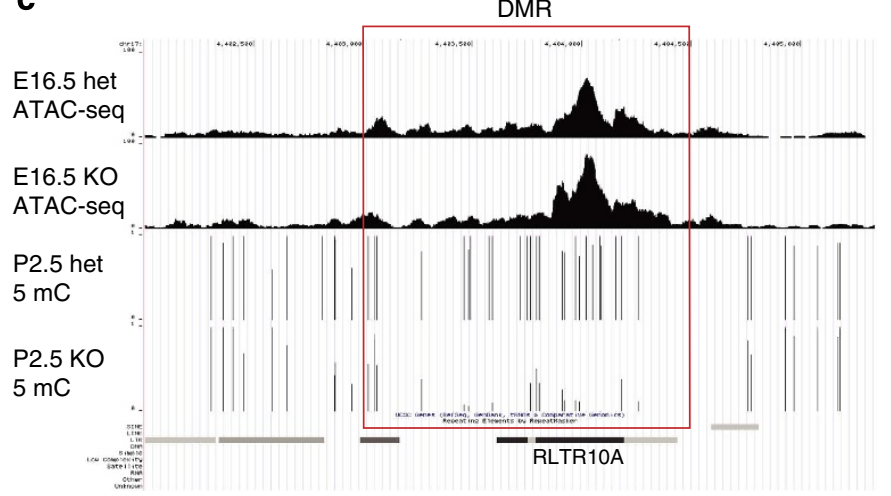

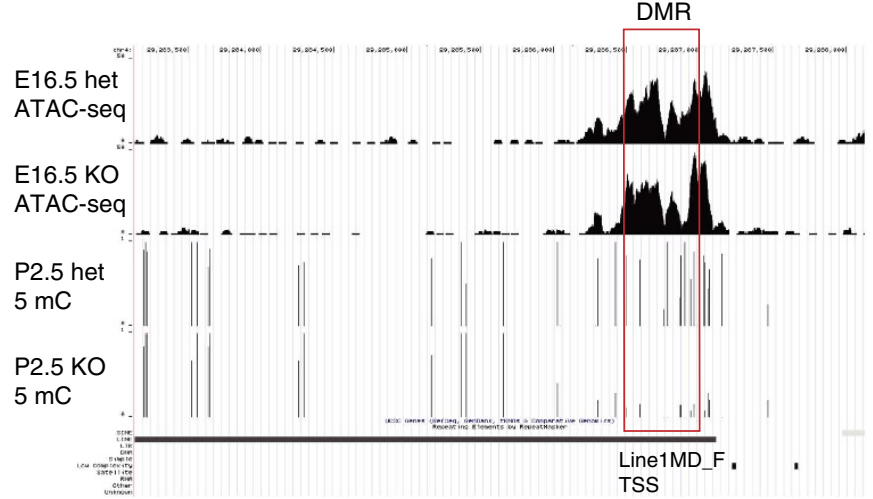

d

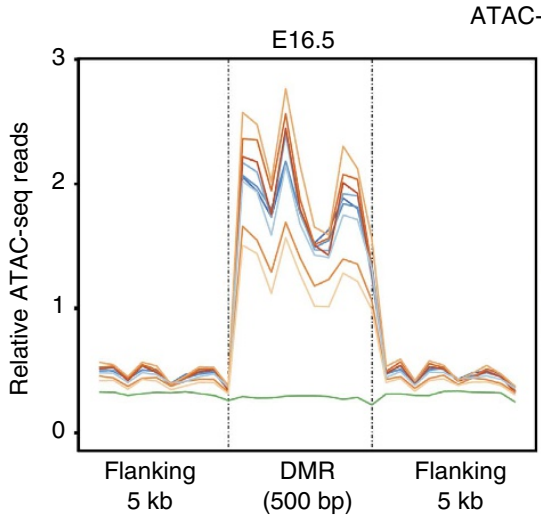

ATAC-seq reads (sorted germ cells)
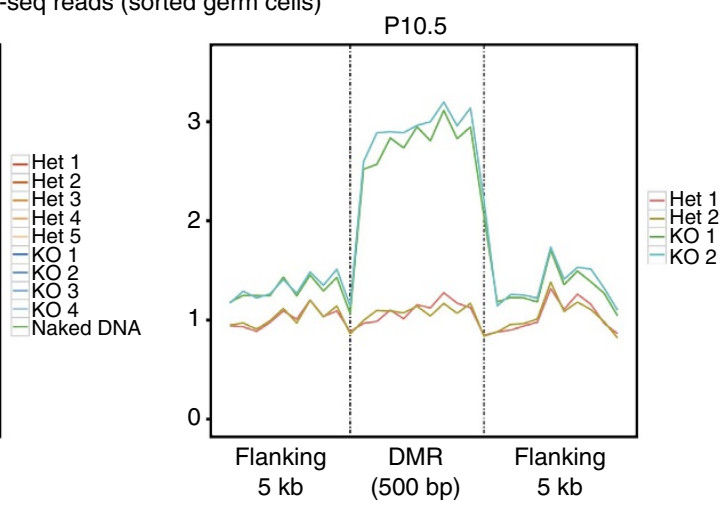

E16.5 KO germ cells

P10.5 het germ cells

P10.5 KO germ cells
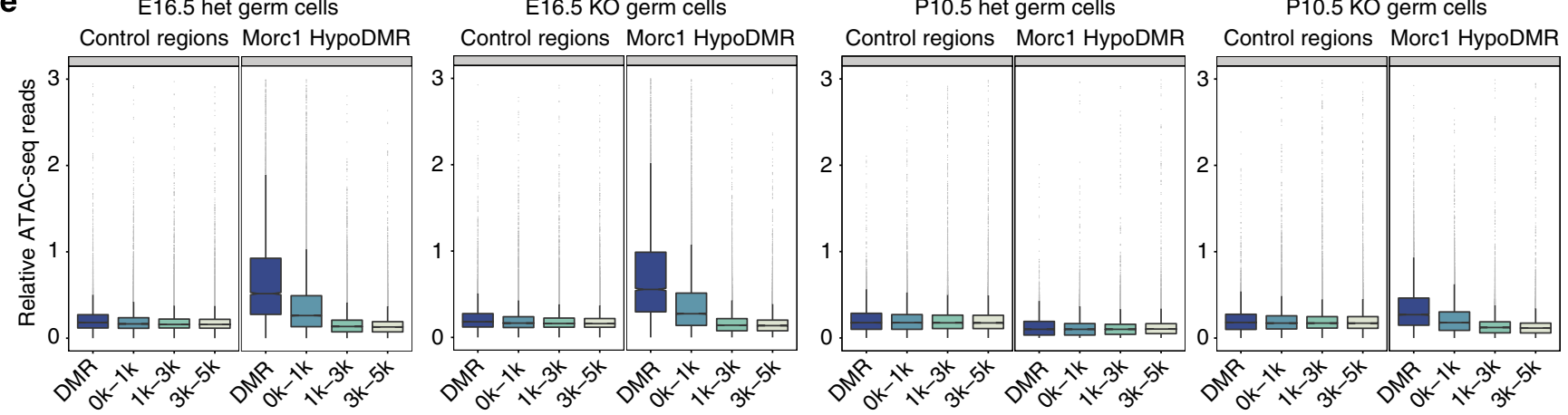

Figure 5 | Hypomethylated regions in Morct ${ }^{\text {tg }}{ }^{\text {tg }}$ correspond to TSS of transposons active in late embryogenesis. (a) H3K 4 me 3 abundance at E16.5 is calculated within regions identified as hypomethylated DMRs (Hypo DMR) in P2.5 Morc $7^{\text {tg }}{ }^{\text {tg }}$ germ cells. (b) Average distributions of uniquely mapping E16.5 RNA-Seq reads (left) and P10.5 RNA-seq reads (right) from individual replicates are plotted over regions identified as hypomethylated at P2.5. (c) Pooled E16.5 ATAC-seq reads are plotted relative to methylation distribution at two loci with hypomethylated DMRs. Each CG is represented as by a bar, with the height of the bar indicating the frequency with which the CG is methylated. A dot at a position indicates no methylation. At least one read must map to the CG for a bar to appear. (d) ATAC-seq reads from individual replicates at E16.5 (left) and P10.5 (right) are plotted relative to DMRs. (e) ATACseq read abundance at DMRs and adjacent regions is represented as a boxplot, with each DMR constituting one point in the boxplot. For a-e, DMRs refer to regions hypomethylated in Morc $1^{\mathrm{tg} / \mathrm{tg}}$ germ cells at P2.5. 
Although we have revealed a critical role for MORC1 in transposon silencing, the actual mechanism by which MORC1 promotes DNA methylation in the male germline is unknown. Our study suggests at least three potential routes by which MORC1 represses transposons and facilitates DNA methylation. One possibility is that MORC1 directly silences transcription, perhaps using its ATPase activity to compact chromatin, thereby reducing $\mathrm{H} 3 \mathrm{~K} 4$ methylation levels at target sites. This silencing would allow for normal de novo methylation by DNMT3L. A second possibility is that MORC1 could recruit an $\mathrm{H} 3 \mathrm{~K} 4$ demethylase, which would similarly promote DNA methylation. Either mechanism agrees with our observation that MORC1hypomethylated DMRs originate from loci with increased $\mathrm{H} 3 \mathrm{~K} 4 \mathrm{me} 3$ at E13.5. A third non-mutually exclusive possibility is that MORC1 directly recruits the DNA methylation machinery to target loci, mediating methylation and silencing.

In conclusion, a robust genome defense system in the male germline is critical to safeguard genome integrity. We have identified a new participant that acts by facilitating DNA methylation of specific repetitive elements classes.

\section{Methods \\ Mice. FVB/N-Morctg/ $+(\mathrm{Tyr}) 1 \mathrm{Az} / \mathrm{J}$ mice $\left(\right.$ Morcl $\left.^{\mathrm{tg}}\right)$ were recovered from cryopre-} servation at the Jackson Laboratory and maintained by intercrossing brothers and sisters in the FVB/N background. Male Morc $^{\mathrm{tg} / \mathrm{tg}}$ mice were viable healthy but infertile, whereas female Morc $1^{\mathrm{t} / \mathrm{tg}}$ mice were viable healthy and fertile. For PCR genotyping, the WT allele was detected as a 347-bp band with the following primers; forward: $5^{\prime}$-ATGCAACTTGAGGGGAAACA-3' and reverse: $5^{\prime}$-GCAGGAG TTATGCGATGTCA- $3^{\prime}$, and the mutant allele was detected as a 244 -bp band with the following primers; forward: $5^{\prime}$-AGTTAGCCGTTATTAGTGGAGAGG- ${ }^{\prime}$ and reverse: $5^{\prime}$-AGAAAGCCTGCCTCAAAACA-3'. PCR conditions involved ten cycles of $94^{\circ} \mathrm{C}, 65^{\circ} \mathrm{C}$ and $68^{\circ} \mathrm{C}$, followed by 28 cycles of $94^{\circ} \mathrm{C}, 50^{\circ} \mathrm{C}$ and $72{ }^{\circ} \mathrm{C}$. For sorting germ cells from E16-5-P2.5, Morcl ${ }^{\mathrm{tg}} / \mathrm{tg}$ females were crossed into the Oct4-IRES-Gfp mixed background. For embryonic staging, timed pregnancies were established and the day a vaginal plug was identified was called embryonic day 0.5 (E0.5). For postnatal time points, the day a litter was first observed was referred to a postnatal day 0.5 ( $\mathrm{P} 0.5)$

All animal experiments were approved by The UCLA Institutional Animal Care and Use Committee, also known as the Chancellor's Animal Research Committee.

Antibodies. Murine Morcl coiled-coil domain (amino acids 788-950), expressed in and purified from bacteria, was provided by Jiamu Du and Dinshaw Patel (Sloan Kettering). Anti-Morc1 antibody was raised in rabbit in collaboration with Rockland Immunochemicals.

Anti-LINE Orflp antibody was provided by Alex Bortvin (Carnegie Institution for Science) and anti-IAP Gaga antibody was provided by Bryan Cullen (Duke).

Immunofluorescence. Whole testes were fixed with 4\% paraformaldehyde, immobilized in paraffin and sectioned. After removal of paraffin, sections were stained at the following antibody concentrations: anti-LINE Orf1p (1:300), anti-IAP Gag (1:300), anti-MORC1 (1:100), anti-VASA (1:100, R\&D Systems AF2030), stained with fluorescent secondary antibody and mounted with DAPI (4',6-diamidino-2-phenylindole). Slides were imaged by Confocal microscopy.

Embryonic germ cell purification. Collection of embryonic testes were performed following institutional approval for appropriate care and use of laboratory animals. Pregnant females were euthanized using $\mathrm{CO}_{2}$ and the embryos removed from the womb and stored on a $10-\mathrm{cm}$ dish filled with chilled $1 \times$ PBS. Testicles were removed from the embryos, placed in an individual 15 -ml falcon tube with $3 \mathrm{ml}$ of $0.25 \%$ Trypsin, with $3 \mu \mathrm{l}$ of DNAse I 1 Unit per $1 \mu$ l (Life Technologies). Testes were incubated for $15 \mathrm{~min}$ at $37^{\circ} \mathrm{C}$. After incubation, the cells were agitated into suspension gently by pipetting. The trypsin was then quenched using $5 \mathrm{ml} \mathrm{DMEM/}$ $10 \%$ fetal bovine serum (Life Technologies). The cells were centrifuged at $278 \mathrm{~g}$ for $5 \mathrm{~min}$ and resuspended in $500 \mu \mathrm{l}$ FACS buffer $(1 \times$ PBS $1 \%$ BSA). 7-Aminoactinomycin $\mathrm{D}$ was added at a 1:50 dilution (BD Biosciences) and the cells strained through BD FACS tubes (Corning) before analysis. Green fluorescent proteinpositive cells were sorted into Buffer RLT (Qiagen) or ATL (Qiagen) for RNA or DNA extraction, respectively.

Postnatal germ cell purification. Pups were euthanized using isoflurane. The testes were removed using tweezers, placed in a $1.5-\mathrm{ml}$ centrifuge tube and chilled on ice. When all testes had been removed, each pair was placed in $1 \mathrm{ml}$ of type IV collagenase (Invitrogen) in an ultra-low-attachment six-well plate (Corning).
All extraneous tissue and the tunica were removed and the seminiferous tubules were teased apart. The samples were then incubated at $37^{\circ} \mathrm{C}$ for $15 \mathrm{~min}$ and centrifuged for $5 \mathrm{~min}$ at $278 \mathrm{~g}$. Testes were then resuspended in $500 \mu \mathrm{l}$ of $0.25 \%$ Trypsin (Life Technologies) and incubated for $5 \mathrm{~min}$ at $37^{\circ} \mathrm{C}$. After the incubation period, the testes were agitated gently into suspension by pipetting. Five hundred microlitres of DMEM $/ 10 \%$ fetal bovine serum was added and the samples were centrifuged for $5 \mathrm{~min}$ at $200 \mathrm{~g}$.

For the P2.5 timepoints, green fluorescent protein-positive cells were sorted as with embryonic time points. To sort germ cells at P10.5, the cells were washed with $1 \mathrm{ml} \mathrm{FACS}$ buffer and then resuspended in $500 \mu \mathrm{l}$ FACS buffer. Cells were then

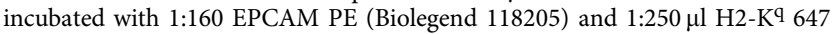
(Biolegend 115106) on ice for $20 \mathrm{~min}$ in the dark, then centrifuged $5 \mathrm{~min}$ at $200 \mathrm{~g}$ and resuspended in $500 \mu \mathrm{l} \mathrm{FACS} \mathrm{buffer.} \mathrm{DAPI} \mathrm{was} \mathrm{added} \mathrm{(1:1,000,} \mathrm{Life}$ Technologies) and the cells were strained through BD FACS tubes (Corning) before analysis. SSC ${ }^{\text {lo }}$ EpCAM $^{\text {hi }} \mathrm{H} 2-\mathrm{K}^{\mathrm{q}-}$ cells were sorted into Buffer RLT or ATL for RNA or DNA extraction, respectively.

qRT-PCR of Morc1. For embryonic samples, gonads were pooled from approximately five to seven mice per time point. RNA was extracted by the TRIzol method and DNase-treated (Qiagen) before complementary DNA conversion (Superscript III, Life Technologies). Quantitative amplification of cDNA was performed in triplicate using SYBR Green quantitation (PCR primers listed below) on a $7900 \mathrm{HT}$ Fast Real Time PCR System (Applied Biosystems).

Rrm2, F: 5'-CCGAGCTGGAAAGTAAAGCG-3'

R: $5^{\prime}$-ATGGGAAAGACAACGAAGCG-3'

Morc Exon 7, F: 5'-GACCCGCAGAAGTTCTTCA-3'

R: 5'-TGCTGCATCAATTCAGCTTC-3'.

RNA preparation. RNA for was extracted from whole testes or cells using the RNeasy Micro Kit (Qiagen 74004). The material was quantified using a Nanodrop ND-1000 (Nanodrop) for RNA from whole testis or the Qubit RNA High Sensitivity Assay (Life Technologies) for RNA from sorted germ cells. RNA quality for material from whole testis was assessed by gel electrophoresis and visualization of the $28 \mathrm{~S}$ and $18 \mathrm{~S}$ rRNA bands.

DNA preparation. DNA for bisulfite sequencing was extracted using the QiaAMP DNA Micro kit (Qiagen) and quantified using the Qubit dsDNA High Sensitivity Kit (Life Technologies)

qRT-PCR of retrotransposons. qRT-PCR for retrotransposons was conducted using published primer sets ${ }^{45}$. One microgram total RNA was treated with DNAse I Amplification Grade (Life Technologies) and converted to cDNA using SuperScript II Reverse transcriptase and random hexamers as primer (Life Technologies). The samples were digested with RNAse $\mathrm{H}$ in accordance with manufacturer's protocol. RT-PCR was then performed using iQ SYBR Green Mastermix (BioRad) with $750 \mathrm{nM}$ concentration of each primer. The samples was amplified (PCR programme: $95^{\circ} \mathrm{C} 10: 00,50 \mathrm{x}\left(95^{\circ} \mathrm{C} 30\right.$ s, $55^{\circ} \mathrm{C} 30$ s, $72^{\circ} \mathrm{C} 30$ s) $)$ with detection of PCR product after each elongation step and determination of melting temperature after the completion of PCR. The reaction was performed using an Agilent Technologies Mx3005p qPCR System (Stratagene). Upregulation of transposon transcript in the mutant is estimated using difference of squares with glyceraldehyde 3-phosphate dehydrogenase as a control.

RNA-seq library preparation. RNA from whole testes was processed for sequencing using a TruSeq RNA Sample Preparation Kit v2 (Illumina) with $250 \mathrm{ng}-2 \mu \mathrm{g}$ total RNA as starting material. Mutant and controls were always matched for starting RNA content. RNA from sorted germ cell was processed using the Ovation Human FFPE RNA-Seq Multiple kit (Nugen) using custom primers for depletion of murine rRNA provided by the manufacturer, using $10 \mathrm{ng}$ of total RNA. Each library was prepared using RNA from one individual mouse.

Small-RNA isolation and library preparation. Total RNA was isolated from embryonic testes using Ribozol. Thirty micrograms of total RNA was loaded on $12 \%$ urea-polyacrylamide (PAA) gel. The 19-30 nt fraction was excised and snapfrozen in liquid nitrogen in $400 \mu \mathrm{l} 0.4 \mathrm{M} \mathrm{NaCl}$. RNA was eluted from the gel overnight at $16^{\circ} \mathrm{C}$ while shaking at 1,000 r.p.m. and then precipitated with $3 \mathrm{vol}$ absolute ethanol. Pre-adenylated $3^{\prime}$-linker (/5'Phos/TGGAATTCTCGGGTGCC AAGGAACTC/ $/ 3^{\prime} \mathrm{ddC} / ; 5^{\prime}$-DNA adenylation kit, NEB) was ligated to RNA overnight at $4{ }^{\circ} \mathrm{C}$ using Truncated RNA Ligase 2 (NEB). Ligation reactions were loaded onto $10 \%$ urea-PAGE, the $45-56 \mathrm{nt}$ fraction was excised and nucleic acids extracted as above. $5^{\prime}$-Linker $\left(5^{\prime}\right.$-rGrUrUrCrArGrArGrUrUrCrUrArCrArGrUrCrCrGrAr CrGrArUrC- $3^{\prime}$ ) was ligated to the samples using RNA Ligase 1 (NEB) overnight at $4{ }^{\circ} \mathrm{C}$. Ligation reactions were loaded on $10 \%$ Urea-PAA gel, $72-83 \mathrm{nt}$ fraction was excised and nucleic acids extracted as above. Extracted samples were reverse transcribed (primer sequence: $5^{\prime}$-GGAGTTCCTTGGCACCCGAGA- $3^{\prime}$ ) and library amplified by PCR using standard Illumina primers. Final libraries were excised from the agarose gel and sequenced. 
Bisulfite library preparation. Libraries were prepared using the Ovation Ultralow Methyl-Seq Library System (Nugen). Five to $25 \mathrm{ng}$ DNA was used as starting material. Matched mutant and control samples always contained identical quantities of DNA. Unmethylated Lambda phage DNA (NEB) was spiked in at $0.5 \%$ input DNA quantity to determine conversion efficiency, which was consistently $>98 \%$. Each library was prepared using DNA from one individual mouse.

ChIP sequencing. The ChIP sequencing (ChIP-seq) protocol was adapted from published sources ${ }^{28}$. FACS-sorted cells from an individual mouse were diluted to $292 \mu \mathrm{l}$ with $1 \times$ PBS at room temperature. Formaldehyde (Sigma) was added to a final concentration of $1 \%$ and the sample was incubated for $10 \mathrm{~min}$ at room temperature with rocking. One molar glycine was then added to yield a final concentration of $0.14 \mathrm{M}$ and the samples were quenched $30 \mathrm{~min}$ with rocking. Cells were then spun at $425 \mathrm{~g}$ for $10 \mathrm{~min}$ at room temperature. The cell pellet was flash frozen.

After thawing, the cells were resuspended in $200 \mu \mathrm{l}$ lysis buffer $(50 \mathrm{mM}$ Tris-Cl pH 8.0, 20 mM EDTA pH 8.0, 1\% SDS, $1 \times$ Complete Protease Inhibitor (Roche)) and incubated on ice for $10 \mathrm{~min}$. Samples were then subjected to a 9-min disruption using a Bioruptor on 'High' setting, with $30 \mathrm{~s} / 30 \mathrm{~s}$ off disruption (hence, $4.5 \mathrm{~min}$ of disruption in total). Samples were spun at $14,000 \mathrm{~g}$ for $10 \mathrm{~min}$, to remove insoluble material. The soluble sample was diluted to $500 \mu \mathrm{l}$ with dilution buffer (16.7 mM Tris pH 8, 0.01\% SDS, 1.1\% TritonX-100, $1.2 \mathrm{mM}$ EDTA, $167 \mathrm{mM} \mathrm{NaCl}$ ) and $10 \%$ of material was saved as input. Sample was precleared with $30 \mu$ Protein A Dynabeads (Life Technologies) and preincubated for $1 \mathrm{~h}$. The cleared material was incubated with $1 \mu \mathrm{l}$ anti-H3K4me3 antibody (Millipore 04-745) overnight.

The samples were incubated with $30 \mu \mathrm{l}$ Protein A Dynabeads and the precipitated material was recovered with a magnet. The beads were washed $2 \times$ for 4 min with Buffer A (50 mM HEPES pH 7.9, $1 \%$ Triton X-100, 0.1\% Deoxycholate, $1 \mathrm{mM}$ EDTA, $140 \mathrm{mM} \mathrm{NaCl}), 2 \times$ for $4 \mathrm{~min}$ with Buffer B (50 mM HEPES $\mathrm{pH} 7.9$, $0.1 \%$ SDS, $1 \%$ Triton X-100, $0.1 \%$ Deoxycholate, $1 \mathrm{mM}$ EDTA, $500 \mathrm{mM} \mathrm{NaCl}$ ) and $2 \times$ for 4 min with $10 \mathrm{mM}$ Tris $/ 1 \mathrm{mM}$ EDTA. Bound material was eluted with $100 \mu \mathrm{l}$ elution buffer ( $50 \mathrm{mM}$ Tris $\mathrm{pH} 8.0,1 \mathrm{mM}$ EDTA, $1 \% \mathrm{SDS}$ ) at $65^{\circ} \mathrm{C}$ for $10 \mathrm{~min}$ and then eluted a second time with $150 \mu \mathrm{l}$ elution buffer.

The input samples were thawed and diluted with $200 \mu \mathrm{l}$ buffer. Cross-linking of $\mathrm{ChIP}$ and input samples was reversed by incubating $16 \mathrm{~h}$ at $65^{\circ} \mathrm{C}$. Samples were cooled and treated with $1.5 \mu \mathrm{l}$ of $10 \mathrm{mg} \mathrm{ml}^{-1}$ RNaseA (PureLink RNAse A, Invitrogen 12091-021) for $30 \mathrm{~min}$ at $37^{\circ} \mathrm{C}$. One hundred micrograms of Proteinase $\mathrm{K}$ was then added and the samples treated for $2 \mathrm{~h}$ at $56^{\circ} \mathrm{C}$. The samples were then purified using a Qiagen Minelute kit.

Samples were amplified by a SeqPlex DNA Amplification kit (Sigma) and then converted to libraries using an Ovation Rapid Library kit.

ATAC-seq library construction. Libraries were generated using a method adapted from published protocol ${ }^{30}$. Briefly, FACS-collected cells from individual mice were spun at $500 \mathrm{~g}$ for $5 \mathrm{~min}$ at $4{ }^{\circ} \mathrm{C}$. Cells were resuspended in $50 \mu \mathrm{l}$ lysis buffer $(10 \mathrm{mM}$ Tris pH 7.4, $10 \mathrm{mM} \mathrm{NaCl}, 3 \mathrm{mM} \mathrm{MgCl}_{2}, 0.1 \% \mathrm{NP} 40,1 \times$ Complete Protease Inhibitor(Roche) ) and spun at $500 \mathrm{~g}$ for $10 \mathrm{~min}$. at $4{ }^{\circ} \mathrm{C}$ to collect nuclei. The nuclei were resuspended in $50 \mu \mathrm{l}$ Transposase reaction $(25 \mu \mathrm{l} 2 \times$ Tagmentation buffer, $22.5 \mu \mathrm{l}$ water, $2.5 \mu \mathrm{l} \operatorname{Tn} 5 \mathrm{Transposase}$ enzyme) and reacted for $30 \mathrm{~min}$ at $37^{\circ} \mathrm{C}$ on a PCR machine. The material was purified using a Qiagen MinElute protocol, eluting with $14 \mu \mathrm{l}$ EB (Qiagen).

To amplify ATAC-seq libraries from the treated material, we amplified using the Ad1 primer below and a different Ad2 primer for each sample, which functions as a barcode

Ad1: 5'-AATGATACGGCGACCACCGAGATCTACACTCGTCGGCAGCG

TCAGATGTG-3'

Ad2.1_TAAGGCGA: 5'-CAAGCAGAAGACGGCATACGAGATTCGCCTT AGTCTCGTGGGCTCGGAGATGT-3'

Ad2.2_CGTACTAG: 5'-CAAGCAGAAGACGGCATACGAGATCTAGTACG GTCTCGTGGGCTCGGAGATGT-3'

Ad2.3_AGGCAGAA: 5'-CAAGCAGAAGACGGCATACGAGATTTCTGCCT GTCTCGTGGGCTCGGAGATGT-3'

Ad2.4_TCCTGAGC: 5'-CAAGCAGAAGACGGCATACGAGATGCTCAGG

AGTCTCGTGGGCTCGGAGATGT-3'

Ad2.5 GGACTCCT: 5'-CAAGCAGAAGACGGCATACGAGATAGGAGTCC

GTCTCGTGGGCTCGGAGATGT-3'

Ad2.6_TAGGCATG: 5'-CAAGCAGAAGACGGCATACGAGATCATGCC

TAGTCTCGTGGGCTCGGAGATGT- $3^{\prime}$

Ad2.7_CTCTCTAC: 5'-CAAGCAGAAGACGGCATACGAGATGTAGAGAG

GTCTCGTGGGCTCGGAGATGT-3'

Ad2.8 CAGAGAGG: 5'-CAAGCAGAAGACGGCATACGAGATCCTCTCTG

GTCTCGTGGGCTCGGAGATGT-3'

Ad2.9_GCTACGCT: 5'-CAAGCAGAAGACGGCATACGAGATAGCGTAGC

GTCTCGTGGGCTCGGAGATGT-3'

Ad2.10_CGAGGCTG: 5' -CAAGCAGAAGACGGCATACGAGATCAGCCTC GGTCTCGTGGGCTCGGAGATGT- $3^{\prime}$

The eluted material was amplified in $50 \mu \mathrm{l}$ volume using $1.25 \mu \mathrm{M}$ primer concentration and a $1 \times$ concentration NEBNext High-Fidelity Master-Mix (NEB) (programme: $72{ }^{\circ} \mathrm{C} 5: 00,98^{\circ} \mathrm{C} 30 \mathrm{~s}, 5 \times\left(98^{\circ} \mathrm{C} 10 \mathrm{~s}, 63^{\circ} \mathrm{C} 30 \mathrm{~s}, 72^{\circ} \mathrm{C} 1 \mathrm{~min}\right), 4^{\circ} \mathrm{C}$ hold). After these five cycles of amplification, the tube was kept on ice.
A 5- $\mu \mathrm{l}$ aliquot was then removed and used to perform a $15-\mu \mathrm{l}$ side reaction with identical concentrations of primer and enzyme as above, except that $0.6 \times$ SYBR Green (Invitrogen S-7563) is included to monitor amplification. This side reaction was amplified on a Stratagene Mx3005p qPCR (Agilent) system with the following amplification conditions $\left(98^{\circ} \mathrm{C} 30 \mathrm{~s}, 20 \times\left(98^{\circ} \mathrm{C} 10 \mathrm{~s}, 63^{\circ} \mathrm{C} 30 \mathrm{~s}, 72^{\circ} \mathrm{C} 1: 100\right)\right.$ ) The number of additional cycles ' $N$ ' required to reach one-fourth maximum fluorescence was observed. The purpose of this side reaction was to minimize the number of PCR cycles required used to generate the libraries, as length and GC bias increases with more amplification The remaining $45 \mu$ of the reaction was then further amplified $\left(98^{\circ} \mathrm{C} 30 \mathrm{~s}, N \times\left(98^{\circ} \mathrm{C} 10 \mathrm{~s}, 63^{\circ} \mathrm{C} 30 \mathrm{~s}, 72^{\circ} \mathrm{C} \mathrm{1:00),} 4^{\circ} \mathrm{C}\right)\right.$ and the libraries were puried by a Qiagen MinElute kit eluting with $20 \mu \mathrm{l}$ volume. Libraries were visualized by running on a 5\% TBE gel and imaged by incubating for $20 \mathrm{~min}$ in $1 \times$ SYBR Green $/ 1 \times$ TBE. Libraries quantified using the KAPA Library Quantification Kit (Kapa Biosystems).

RNA-seq analysis. For all analyses, reads were trimmed to $50 \mathrm{bp}$ and those mapping to ribosomal RNA (GenBank identifiers: 18S NR_003278.3, 28S NR_003279.1, 5S D14832.1, 5.8S K01367.1) by up to three mismatches were discarded.

Analysis on repeat families. Reads were then mapped to the $\mathrm{mm} 9$ genome allowing no mismatches and keeping reads that map up to 10,000 sites in the genome using Bowtie ${ }^{46}$. Each mapping read was assigned a score of $1 / n$, where $n$ is the number of sites in the genome the read mapped to. Repeats were obtained from RepeatMasker. Expression values for each repeat family was calculated by adding the scores contained within the repeat body, divided by the total million reads mapped and average length $(\mathrm{kb})$ of repeats within the family.

Analysis on individual genes and repeats. Reads were then mapped to known $\mathrm{mm} 9$ gene and repeat annotations by allowing up to two mismatches and only retaining reads that mapped to one location. When reads did not map to the annotated genes and repeats, the reads were mapped to the $\mathrm{mm} 9$ genome. Number of reads mapping to genes and repeats were determined by using HTSeq (doi: 10.1101/002824) using default parameters. Expression values were calculated as reads per kilobase of exons per million mapping reads. Differential gene and repeat expression was determined by using DESeq ${ }^{47}$, by using default parameters.

Whole-genome bisulfite sequencing. Reads were split into $50 \mathrm{bp}$ reads before mapping. Reads were mapped to the $\mathrm{mm} 9$ genome as well as the lambda genome using BS seeker2 (ref. 48) using default parameters. Methylation levels were determined by $\# \mathrm{C} /(\# \mathrm{C}+\# \mathrm{~T})$. For identifying DMRs, the genome was tiled into 500 bp bins and CG methylation levels in knockout and control were compared within each bin. Bins that had a methylation level difference of $50 \%$ as well as a false discovery rate $<0.05$ calculated by Fisher's exact test corrected by the Benjamini-Hochberg procedure were selected. Finally, DMRs containing at least four cytosines in CG contexts, each covered by at least four reads were retained. Control regions were defined completely randomly, except that control regions have (1) exact same coverage of cytosines in CG contexts as the Morcl ${ }^{+}$tt data within DMRs; (2) WT CG methylation levels are similar as the $\mathrm{Morcl}^{+/ \operatorname{tg}}$ data within DMRs ( $<5 \%)$. (3) same number of regions per chromosome as DMRs. We defined genes as associated with DMRs when the TSS of an Ensembl transcript model was within $1 \mathrm{~kb}$ of a DMR.

To align to Repeat consensus sequences, the RepBase consensus sequences for 30 repetitive elements (B1_SINE, ERVB7 1-I MM (EtnERV2/MusD), IAP-d, IAPEY3_I, IAPEY_I, IAPEY_LTR, IAPEY3_LTR, IAPEZI, IAPLTR1_Mm, IAPLTR2_Mm, IAPLTR3, IAPLTR3_I, IAPLTR4, IAPLTR4-I, L1MdA_I, L1MdF_I, L1MdGf_I, L1MdTf_I, First 234 bases of GSAT_MM (Major_satellite), MMERGLN_I, MMERGLN_LTR, MERVL, MERVL_LTR, First 120 bases of SATMIN (Minor satellite), MMERVK10C, RLTR10C, RLTR27_MM RLTR6_MM, RLTR6I_MM, RLTRETN_MM and RSINE1 were combined into a microgenome. Then, whole-genome bisulfite sequencing reads were mapped to the microgenome using $\mathrm{BSMAP}^{49}$, accepting uniquely mapping reads only $[-\mathrm{w} 1]$, mapping to two forward possible strands $[-\mathrm{n} 0]$ and allowing 2 mismatches $[-\mathrm{v}$ 2]. Methylation levels were determined by $\# \mathrm{C} /(\# \mathrm{C}+\# \mathrm{~T})$. The methylation levels at each CG site was calculated.

Small RNA sequencing. Sequence adaptors were removed using a customdesigned dynamic programming algorithm that recognizes both exact and inexact matches, and the trimmed reads were aligned to the $\mathrm{mm} 9$ genome following a custom suffix array-based procedure ${ }^{50}$. Reads with lengths $>24$ nt were considered for piRNA analysis. Based on alignment coordinates, the reads were annotated as derived from exons, introns, transposons and other repeats according to the genome annotation obtained via UCSC Genome Bioinformatics ${ }^{51}$. Reads that had multiple valid alignments were annotated based on ten alignments selected at random, and the majority annotation was assigned as the final annotation. In case of ties, annotation was picked based on a fixed hierarchy principle ${ }^{50}$. Sense or antisense annotation was assigned to piRNA reads with respect to the strandedness of an underlying genomic feature. If a piRNA read contained $\mathrm{U}$ in position 1 , such piRNA was considered as primary, while the presence of $\mathrm{A}$ in position 10 defined secondary piRNAs. 
ChIP-seq analysis. Previously published ChIP-seq data ${ }^{28}$ was obtained from GSE38165 in Gene Expression Omnibus. Reads were mapped to the $\mathrm{mm} 9$ genome using Bowtie by allowing up to two mismatches and only retaining reads that mapped to one location in the genome. Reads mapping to the same location were collapsed into one read. For all analyses, the data were normalized to total number of mapping reads in the library

ATAC-seq analysis. Data were collected using $50 \mathrm{bp}$ paired end sequencing on a HiSeq. In keeping with established methodologies ${ }^{30}$, reads were aligned to $\mathrm{mm} 9$ using Bowtie ${ }^{52}$ with the parameters $-\mathrm{X} 2000$ and $-\mathrm{m} 1$. The $-\mathrm{X} 2000$ parameter allows the fragments $<2 \mathrm{~kb}$ to align and only unique aligning reads were collected $(-\mathrm{m} 1)$. Duplicated reads were removed with samtools (rmdup function) ${ }^{53}$. Previous results show that for $\operatorname{Tn} 5$ transposase, the transposon binds as a dimer and insert two adaptors separated by $9 \mathrm{bp}^{54}$. Thus, all reads aligned to the positive strands were offset by $+4 \mathrm{bp}$ and all reads aligned to the negative strands were offset by $-5 \mathrm{bp}$.

\section{References}

1. Moissiard, G. et al. MORC family ATPases required for heterochromatin condensation and gene silencing. Science 336, 1448-1451 (2012).

2. Lorkovic, Z. J., Naumann, U., Matzke, A. J. \& Matzke, M. Involvement of a GHKL ATPase in RNA-directed DNA methylation in Arabidopsis thaliana. Curr. Biol. 22, 933-938 (2012).

3. Hruz, T. et al. Genevestigator $\mathrm{v} 3$ : a reference expression database for the meta-analysis of transcriptomes. Adv. Bioinformatics 2008, 420747 (2008).

4. Watson, M. L. et al. Identification of morc (microrchidia), a mutation that results in arrest of spermatogenesis at an early meiotic stage in the mouse. Proc. Natl Acad. Sci. USA 95, 14361-14366 (1998).

5. Inoue, N. et al. New gene family defined by MORC, a nuclear protein required for mouse spermatogenesis. Hum. Mol. Genet. 8, 1201-1207 (1999).

6. Popp, C. et al. Genome-wide erasure of DNA methylation in mouse primordial germ cells is affected by AID deficiency. Nature 463, 1101-1105 (2010).

7. Seisenberger, S. et al. The dynamics of genome-wide DNA methylation reprogramming in mouse primordial germ cells. Mol. Cell 48, 849-862 (2012).

8. Kobayashi, H. et al. High-resolution DNA methylome analysis of primordial germ cells identifies gender-specific reprogramming in mice. Genome Res. 23, 616-627 (2013).

9. Kaneda, M. et al. Essential role for de novo DNA methyltransferase Dnmt3a in paternal and maternal imprinting. Nature 429, 900-903 (2004).

10. Bourc'his, D. \& Bestor, T. H. Meiotic catastrophe and retrotransposon reactivation in male germ cells lacking Dnmt3L. Nature 431, 96-99 (2004).

11. Webster, K. E. et al. Meiotic and epigenetic defects in Dnmt3L-knockout mouse spermatogenesis. Proc. Natl Acad. Sci. USA 102, 4068-4073 (2005).

12. Carmell, M. A. et al. MIWI2 is essential for spermatogenesis and repression of transposons in the mouse male germline. Dev. Cell 12, 503-514 (2007).

13. Shoji, M. et al. The TDRD9-MIWI2 complex is essential for piRNA-mediated retrotransposon silencing in the mouse male germline. Dev. Cell 17, 775-787 (2009).

14. Iyer, L. M., Abhiman, S. \& Aravind, L. MutL homologs in restriction-modification systems and the origin of eukaryotic MORC ATPases. Biol. Direct 3, 8 (2008).

15. Wernig, M. et al. In vitro reprogramming of fibroblasts into a pluripotent ES-cell-like state. Nature 448, 318-324 (2007).

16. Vincent, J. J. et al. Single cell analysis facilitates staging of Blimp1-dependent primordial germ cells derived from mouse embryonic stem cells. PLOS ONE 6, e28960 (2011)

17. Small, C. L., Shima, J. E., Uzumcu, M., Skinner, M. K. \& Griswold, M. D. Profiling gene expression during the differentiation and development of the murine embryonic gonad. Biol. Reprod. 72, 492-501 (2005).

18. Hata, K., Kusumi, M., Yokomine, T., Li, E. \& Sasaki, H. Meiotic and epigenetic aberrations in Dnmt3L-deficient male germ cells. Mol. Reprod. Dev. 73, 116-122 (2006)

19. Aravin, A. A. et al. A piRNA pathway primed by individual transposons is linked to de novo DNA methylation in mice. Mol. Cell 31, 785-799 (2008).

20. Bellve, A. R., Millette, C. F., Bhatnagar, Y. M. \& O'Brien, D. A. Dissociation of the mouse testis and characterization of isolated spermatogenic cells. J. Histochem. Cytochem. 25, 480-494 (1977).

21. Oatley, J. M. \& Brinster, R. L. Spermatogonial stem cells. Methods Enzymol. 419, 259-282 (2006).

22. Niles, K. M., Chan, D., La Salle, S., Oakes, C. C. \& Trasler, J. M. Critical period of nonpromoter DNA methylation acquisition during prenatal male germ cell development. PLoS ONE 6, e24156 (2011).

23. Tomizawa, S. et al. Dynamic stage-specific changes in imprinted differentially methylated regions during early mammalian development and prevalence of non-CpG methylation in oocytes. Development 138, 811-820 (2011).

24. Watanabe, T. et al. Role for piRNAs and noncoding RNA in de novo DNA methylation of the imprinted mouse Rasgrf1 locus. Science 332, 848-852 (2011).

25. Chotalia, M. et al. Transcription is required for establishment of germline methylation marks at imprinted genes. Genes Dev. 23, 105-117 (2009).
26. Sookdeo, A., Hepp, C. M., McClure, M. A. \& Boissinot, S. Revisiting the evolution of mouse LINE-1 in the genomic era. Mobile DNA 4, 3 (2013).

27. Leung, D. C. \& Lorincz, M. C. Silencing of endogenous retroviruses: when and why do histone marks predominate? Trends Biochem. Sci. 37, 127-133 (2012)

28. Ng, J. H. et al. In vivo epigenomic profiling of germ cells reveals germ cell molecular signatures. Dev. Cell 24, 324-333 (2013).

29. Ooi, S. K. et al. DNMT3L connects unmethylated lysine 4 of histone $\mathrm{H} 3$ to de novo methylation of DNA. Nature 448, 714-717 (2007).

30. Buenrostro, J. D., Giresi, P. G., Zaba, L. C., Chang, H. Y. \& Greenleaf, W. J. Transposition of native chromatin for fast and sensitive epigenomic profiling of open chromatin, DNA-binding proteins and nucleosome position. Nat. Methods 10, 1213-1218 (2013).

31. Kuramochi-Miyagawa, S. et al. Mili, a mammalian member of piwi family gene, is essential for spermatogenesis. Development 131, 839-849 (2004).

32. Aravin, A. A., Sachidanandam, R., Girard, A., Fejes-Toth, K. \& Hannon, G. J. Developmentally regulated piRNA clusters implicate MILI in transposon control. Science 316, 744-747 (2007).

33. Watanabe, T. et al. MITOPLD is a mitochondrial protein essential for nuage formation and piRNA biogenesis in the mouse germline. Dev. Cell 20, 364-375 (2011).

34. Frost, R. J. et al. MOV10L1 is necessary for protection of spermatocytes against retrotransposons by Piwi-interacting RNAs. Proc. Natl Acad. Sci. USA 107, 11847-11852 (2010)

35. Zheng, K. et al. Mouse MOV10L1 associates with Piwi proteins and is an essential component of the Piwi-interacting RNA (piRNA) pathway. Proc. Natl Acad. Sci. USA 107, 11841-11846 (2010).

36. Soper, S. F. et al. Mouse maelstrom, a component of nuage, is essential for spermatogenesis and transposon repression in meiosis. Dev. Cell 15, 285-297 (2008).

37. Aravin, A. A. et al. Cytoplasmic compartmentalization of the fetal piRNA pathway in mice. PLoS Genet. 5, e1000764 (2009).

38. Saxe, J. P., Chen, M., Zhao, H. \& Lin, H. Tdrkh is essential for spermatogenesis and participates in primary piRNA biogenesis in the germline. EMBO J. 32, 1869-1885 (2013).

39. Tanaka, S. S. et al. The mouse homolog of Drosophila vasa is required for the development of male germ cells. Genes Dev. 14, 841-853 (2000).

40. Kuramochi-Miyagawa, S. et al. MVH in piRNA processing and gene silencing of retrotransposons. Genes Dev. 24, 887-892 (2010).

41. Molaro, A. et al. Two waves of de novo methylation during mouse germ cell development. Genes Dev. 28, 1544-1549 (2014).

42. Ollinger, R. et al. Deletion of the pluripotency-associated Tex19.1 gene causes activation of endogenous retroviruses and defective spermatogenesis in mice. PLoS Genet. 4, e1000199 (2008).

43. Tarabay, Y. et al. The mammalian-specific Tex19.1 gene plays an essential role in spermatogenesis and placenta-supported development. Hum. Reprod. 28, 2201-2214 (2013).

44. Reichmann, J. et al. Microarray analysis of LTR retrotransposon silencing identifies $\mathrm{Hdacl}$ as a regulator of retrotransposon expression in mouse embryonic stem cells. PLoS Comput. Biol. 8, e1002486 (2012).

45. Karimi, M. M. et al. DNA methylation and SETDB1/H3K9me3 regulate predominantly distinct sets of genes, retroelements, and chimeric transcripts in mESCs. Cell Stem Cell 8, 676-687 (2011).

46. Langmead, B., Schatz, M. C., Lin, J., Pop, M. \& Salzberg, S. L. Searching for SNPs with cloud computing. Genome Biol. 10, R134 (2009).

47. Anders, S. \& Huber, W. Differential expression analysis for sequence count data. Genome Biol. 11, R106 (2010).

48. Guo, W. et al. BS-Seeker2: a versatile aligning pipeline for bisulfite sequencing data. BMC Genomics 14, 774 (2013).

49. Xi, Y. \& Li, W. BSMAP: whole genome bisulfite sequence MAPping program. BMC Bioinformatics 10, 232 (2009).

50. Olson, A. J., Brennecke, J., Aravin, A. A., Hannon, G. J. \& Sachidanandam, R. Analysis of large-scale sequencing of small RNAs. Pac. Symp. Biocomput. 13, 126-136 (2008).

51. Karolchik, D. et al. The UCSC Genome Browser database: 2014 update. Nucleic Acids Res. 42, D764-D770 (2014).

52. Langmead, B., Trapnell, C., Pop, M. \& Salzberg, S. L. Ultrafast and memoryefficient alignment of short DNA sequences to the human genome. Genome Biol. 10, R25 (2009).

53. Li, H. et al. The sequence alignment/map format and SAMtools. Bioinformatics 25, 2078-2079 (2009).

54. Adey, A. et al. Rapid, low-input, low-bias construction of shotgun fragment libraries by high-density in vitro transposition. Genome Biol. 11, R119 (2010).

\section{Acknowledgements}

We acknowledge the UCLA BSCRC Flow Cytometry core for flow and FACS assistance, and the UCLA BSCRC High Throughput Sequencing Core. Bryan Cullen provided anti-IAP Gag antibody and Alexander Bortvin provided anti-LINE ORF1p antibody. Jiamu $\mathrm{Du}$ and Dinshaw Patel provided recombinant MORCl coiled-coil domain to raise antibody. 
W.A.P. is supported by the Jane Coffin Childs Memorial Fund for Medical Research. H.S. is an HHMI Fellow of the Damon Runyon Cancer Research Foundation (DRG-2194-14). W.L. is supported by a grant from the Chinese Scholar Council. This work was supported by grants from the NIH (R01 HD058047 and DP2 OD007371A), a Research Award from the Eli and Edythe Broad Center of Regenerative Medicine and Stem Cell Research and the Nogradi Fund, the Searle Scholar and the Packard Fellowship Awards, and an ANR grant ("TranspoFertil"). S.E.J. is an investigator of the Howard Hughes Medical Institute.

\section{Author contributions}

W.A.P., K.N., D.P., N.Z. and S.A.L. managed mice, performed dissections and purified DNA and RNA from materials. W.A.P., D.P. and G.M. generated sequencing libraries. H.S., W.L. and S.M. performed bioinformatics analysis. W.A.P. and S.A.L. performed immunofluorescent staining of target tissue. W.A.P, A.T.C. and S.E.J. wrote the manuscript. D.B., A.A.A., A.T.C. and S.E.J. coordinated research.

\section{Additional information}

Accession codes: Raw sequencing data, including RNA-seq, whole-genome bisulfite-seq, small RNA-seq, ATAC-seq and ChIP-seq, generated for this study have been deposited in the GEO database under accession number GSE63048.
Supplementary Information accompanies this paper at http://www.nature.com/ naturecommunications

Competing financial interests: The authors declare no competing financial interests.

Reprints and permission information is available online at http://npg.nature.com/ reprintsandpermissions/

How to cite this article: Pastor, W. A. et al. MORC1 represses transposable elements in the mouse male germline. Nat. Commun. 5:5795 doi: 10.1038/ncomms6795 (2014).

(c) (i)

This work is licensed under a Creative Commons Attribution 4.0 International License. The images or other third party material in this article are included in the article's Creative Commons license, unless indicated otherwise in the credit line; if the material is not included under the Creative Commons license, users will need to obtain permission from the license holder to reproduce the material. To view a copy of this license, visit http://creativecommons.org/licenses/by/4.0/ 


\section{Erratum: MORC1 represses transposable elements in the mouse male germline}

William A. Pastor, Hume Stroud, Kevin Nee, Wanlu Liu, Dubravka Pezic, Sergei Manakov, Serena A. Lee, Guillaume Moissiard, Natasha Zamudio, Déborah Bourc'his, Alexei A. Aravin, Amander T. Clark $\&$ Steven E. Jacobsen

Nature Communications 5:5795 doi: 10.1038/ncomms6795 (2014); Published 12 Dec 2014; Updated 10 Jul 2015

This Article contains errors in Fig. 2 that were introduced during the production process. The bars of the graph in panel $f$ were inadvertently switched with those of the graph in panel g. The correct version of the figure appears below. 
a

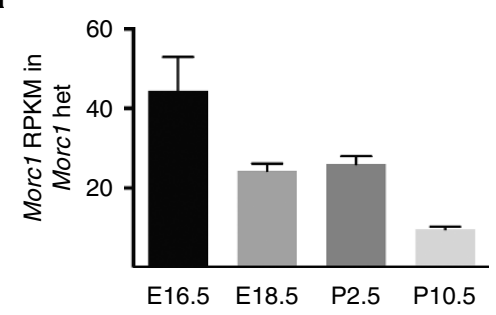

C

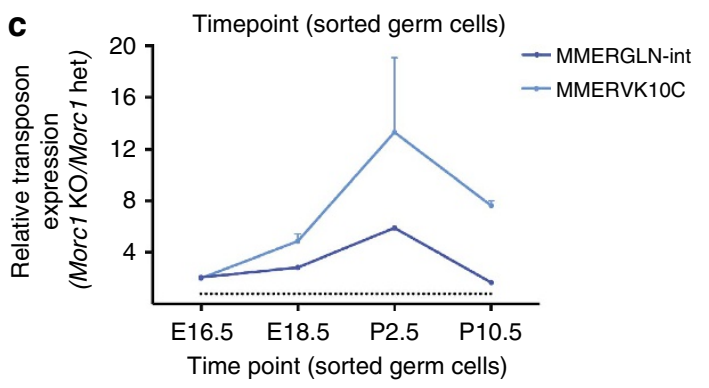

e

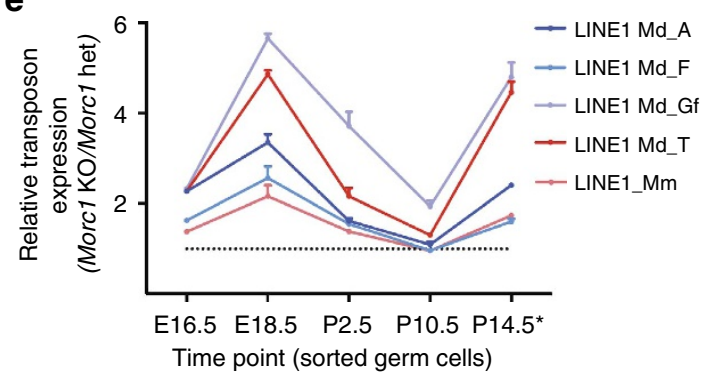

*Whole testis

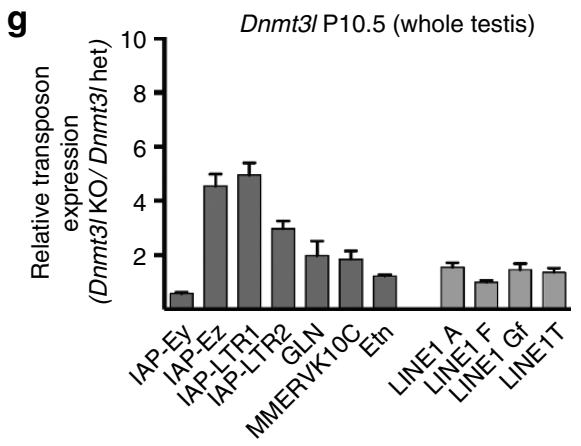

b

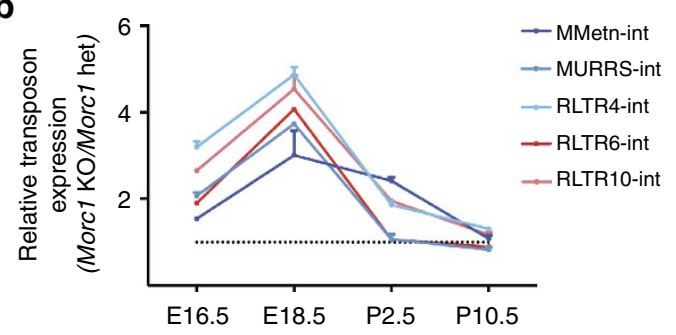

d

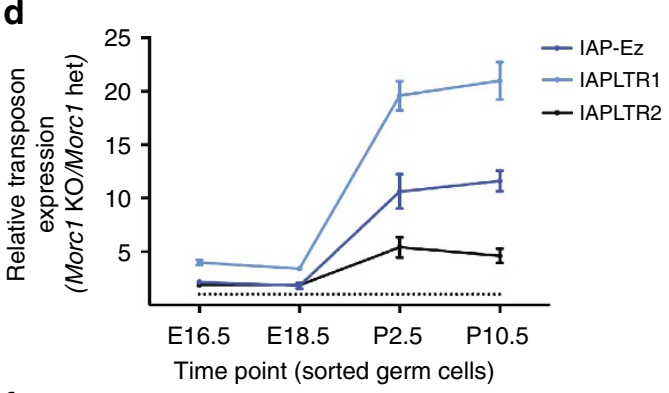

f

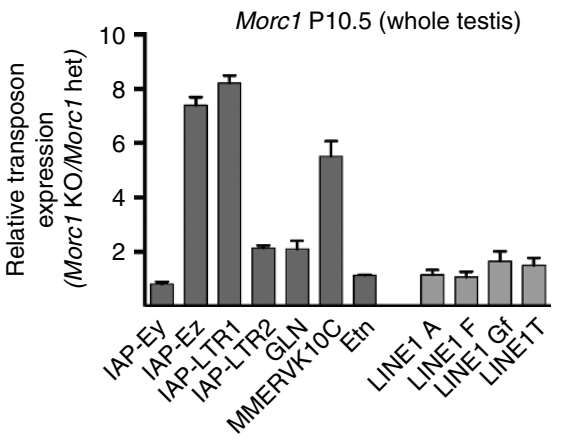

h

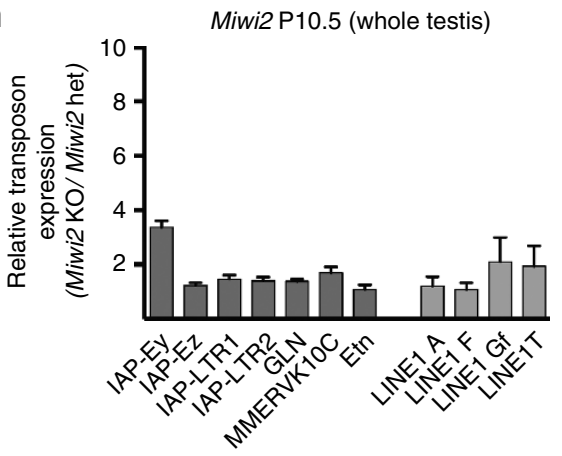

Figure 2 